\title{
A laminated structural finite element for the behavior of large non-linear reinforced concrete structures.
}

\author{
Cuauhtemoc Escudero*, Sergio Oller, Xavier Martinez, Alex H. Barbat \\ Departamento de Resistencia de Materiales y Estructuras en la Ingenieía, Universidad Politécnica de Cataluña, Jordi Girona 1-3, \\ Módulo C1 Campus Norte UPC, Barcelona 08034, Spain
}

\begin{abstract}
In order to correctly predict the kinematics of complex structures, analysis using three-dimensional finite elements (3DFEs) seems to be the best alternative. However, simulation of large multi-layered structures with many plies can be unaffordable with 3DFEs because of the excessive computational cost, especially for non-linear materials. In addition, the discretization of very thin layers can lead to highly distorted FEs carrying numerical issues, therefore, reduced models arise as an affordable solution.

This paper describes a new finite element formulation to perform numerical simulations of laminated reinforced concrete structures. The intention of this work is that the proposed scheme can be applied in the analysis of real-life structures where a high amount of computational resources are needed to fulfill the meshing requirements, hence the resulting formulation has to be a compromise between simplicity and efficiency.

So that, the condensation of a dimension (thickness), mandatory to model three-dimensional structures with twodimensional finite elements (2DFEs), leads to refer all layers contained within such FEs to a plane, which is typically named middle plane or geometrical plane, since its sole function is to serve as a geometrical reference. This work is based on the assumption that the geometrical plane has to be distinguished from a mechanical plane, which is where the resultant stiffness of all layers is contained. It is also assumed in this work that the mechanical plane changes its position due to non-linear response of the component materials.
\end{abstract}

(C) 2015 Published by Elsevier Ltd.

Keywords:

Laminated element, Composite materials, large RC structures, mechanical plane

\section{Introduction}

Current theories that allow the use of two-dimensional FEs to model composite materials, yet powerful, lack the necessary simplicity for their application in complex structures where a large amount of FE is required for a good approximation in the obtained results.

Thus, simpler and more efficient techniques are required for modelling laminated structures, where the three-dimensional description can be reduced to a two-dimensional model by introducing hypotheses on the

\footnotetext{
* Corresponding author.

Email addresses: cuauhtemoc@cimne.upc.edu (Cuauhtemoc Escudero ), sergio.oller@upc.edu (Sergio Oller), xmartinez@cimne.upc.edu (Xavier Martinez), alex . barbat@upc . edu (Alex H. Barbat)
} 
(a)

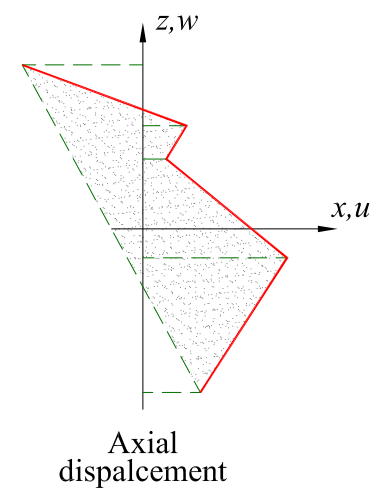

(b)

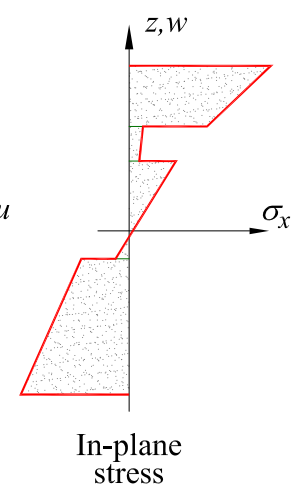

(c)

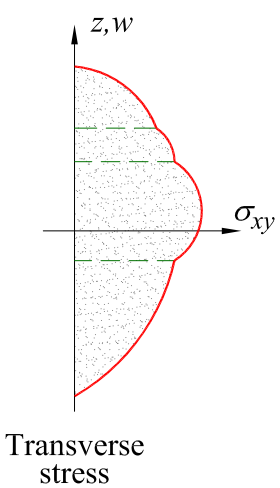

Fig. 1 Continuous zigzag in-plane displacement a), discontinuous in-plane stress b), and continuous transverse stress $c$ ).

displacements and/or on the stresses field, since laminate thickness is at least one order of magnitude lower than in-plane dimensions.

Reference [1] provides an overview of the available theories and finite elements that have been developed for multi-layered, anisotropic, composite plate and shell structures. Multi-scale approaches [2, 3] can also be used to model non-linear multi-layered materials. In this method a macroscopic model is used to obtain the global response of the structure whereas the material behaviour, modelled with a constitutive law, is solved with a microscopic model.

Many reduced approaches have been developed and improved since 19th century. In order to facilitate their classification, they could be distinguished according to [4]:

a) The type of unknown variable chosen, so they could be Displacement Based Theories (DB), StressBased Theories (SB), or if both stress and displacement are considered as unknowns, a Mixed Approach $(\mathrm{MB})$ is obtained.

b) How the unknown variables are described. In this classification it may be an Equivalent Single Layer (ESL) description, where governing equations are written for the whole plate, or a Layer-Wise (LW) description, where each layer is treated independently assuming separate displacement/stress fields within each ply, which leads to write the governing equations for each layer.

From the previous classification, the most basic DB-ESL model is the Classical Theory (CT) [5], whereas an improvement to the CT theory is the First Order Shear Deformation Theory (FSDT) [6] which enhances the CT kinematics by adding shear effects. Although CT and FSDT are excellent alternatives to accurately model homogeneous thin and thick structures, they lead to poor prediction in the cases listed below

- Where component materials have a high level of transverse anisotropy.

- When applied to the analysis of composite laminated with embedded debounding.

- When it is necessary to provide regions with 3-D stresses fields, i.e. $\sigma_{Z} \neq 0$.

- When it is required to capture the so called zig-zag pattern of in-plane displacements (ZZ condition).

- When it is required to satisfy the condition of continuous transverse shear along the thickness direction (TC condition).

The cause is found in the linear thickness distribution of the axial displacement, which does not match the $Z Z$ pattern depicted in figure 1 [4]. 
In order to fulfill the previously listed condition it must be necessary to use either a theory based on 3-D kinematics, or a LW based theory. Although LW theories accurately fulfill both, the ZZ and the TC condition, the number of unknown variables is proportional to the number of analyzed layers. As a result, these models yield not only a high level of accuracy but also to an amount of unknown variables similar to the 3D analysis. For this reason, LW models may result unattractive for simulating large laminated structures with many plies. Therefore, these models should be employed to analyze complex problems where other less expensive approaches fail to give realistic predictions [4].

A special case of LW model where the number of unknowns is independent of the number of analyzed layer is the Zigzag theory (ZZT), which is a good compromise between the accuracy of MB-LW theories and the computational efficiency of DB-ESL models. One of the most important advantages of these theories is that the number of kinematics unknowns are independent of the number of analyzed layers.

Have to be remarked that, the study of the cases where it is mandatory to use a LW description are out of the scope of this work, and the only feasible solution from a computational point of view, which allows to achieve good results, is to adopt a ESL scheme.

That is why it has been proposed a scheme capable of reproducing the bending damage of a laminated material without the need of additional degrees of freedom than the ones listed below.

$$
\boldsymbol{d}=\left\{w_{1} \theta_{x 1} \theta_{y 1} w_{2} \theta_{x 2} \theta_{y 2} w_{3} \theta_{x 3} \theta_{y 3}\right\}^{\mathrm{T}}
$$

This simplification is justified by the fact that stiffness of the simple materials used for reinforced concrete (RC) structures never exceeds an order of magnitude. In addition, in order to avoid shear locking situations [7], the proposed scheme has been implemented using a Discrete Kirchhoff Triangle [8] where the shear transverse strains are postulated to be neglected with respect to other strains.

The paper is organized as follows. We start by presenting the basic framework of the plate theory, and later the proposed modification, to finally detail an implementation into the FE framework. Section 3 describes the application of the proposed scheme to non-linear materials, and consequently, to plates with bending degradation. Finally, in section 4 it is shown the performance of the proposed plate scheme, and are presented some numerical examples; in particular, a linear clamped beam, a linear clamped plate with a notch, a non-linear clamped beam, an unreinforced concrete frame and a reinforced concrete frame.

\section{Basic framework and FE implementation}

\subsection{Geometry and load.}

The plate term is referred to a flat slender body, occupying the domain

$$
\Omega=\left\{(x, y, z) \in \mathbb{R} \mid z \in\left[-\frac{t}{2},+\frac{t}{2}\right], \quad(x, y) \in \mathscr{A} \subset \mathbb{R}^{2}\right\}
$$

where the plane $Z=0$ coincides with the mid-plane (also referred as the geometrical along this work) of the undeformed plate and the transverse dimension, of thickness $t$, is small compared with the other two dimensions.

\subsection{Kinematics}

The infinitesimal kinematic for a plate always assume the following displacement fields:

$$
u(x, y, z)=-z \theta_{x}(x, y), \quad v(x, y, z)=-z \theta_{y}(x, y), \quad w(x, y, z)=w(x, y)
$$

where $u, v$ and $w$ are the displacements along $x, y$ and $z$ axes respectively, and $\theta_{x}$ and $\theta_{y}$ are the rotations of the transverse line elements about the $x$ and $y$ axes. Accordingly, a straight line element, normal to the plate mid-surface in the undeformed configuration could either remain straight and normal according to the classical Kirchhoff plate theory, or not necessarily normal according to Reissner [9] and Mindlin [10], being the difference among such theories the effect of the transverse shear deformation. 

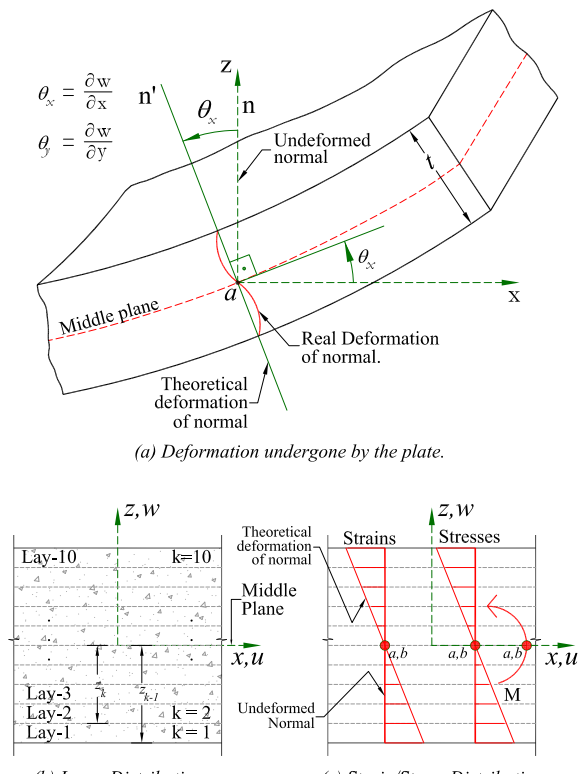

(b) Layer Distribution

(c) Strain/Stress Distribution

Fig. 2 Middle plane deformation for a thin plate.

The use of classical theories for thin and thick plates applied to homogeneous plates is the best alternative. However, when it comes to laminated plates formed by layers made of composite materials, the use of such theories is not sufficient to assess the in-plane deformation fields of their components. That is why this paper proposes a scheme in which the in-plane displacements field $u$ and $v$ are evaluated according to the mechanical and constitutive properties of the constituent materials.

First, let us assume the existence of points $a$ and $b$. Point $a$ is defined as the intersection of the middle plane and the undeformed normal of the plate, whereas point $b$ is defined as the intersection of the theoretical deformation of the normal and the neutral (or mechanical) plane. The purpose of using points $a$ and $b$ is to refer the displacement field $w$ to point $a$, whereas point $b$ will be the reference for rotations $\theta_{x}$ and $\theta_{y}$, consequently, it will be used as the reference for in-plane displacements $u$ and $v$ of the layers within the laminate material. Figures 2.a and 3.a are used as an aid to express this idea. The case when $a=b$ is where the plate is formed by a homogeneous material, as depicted in figure 2 .

Now, let us assume the particular laminate from figure 2.b in its layer 8 has a material stiffener than the rest, leading to the case illustrated in figure 3.b. This is $a \neq b$, and the consequences is that the strains distribution although remain linear, no longer remain symmetrical to the middle plane, also there is a variation in the stress distribution and in the generalized stresses $\hat{\sigma}$, which in figure 3.c are represented using bending moment M. From figure 3.b can also be noticed that at point $b$ (neutral plane) the in-plane displacements are equal to $u=0$ and $v=0$.

The same as for both thick and thin plate theories, the integration of the in-plane stresses $\sigma_{x}, \sigma_{y}$ and $\gamma_{x y}$, and the transverse shears stresses $\gamma_{x z}$ and $\gamma_{y z}$ define the stress resultant per unit length

$$
\begin{gathered}
\hat{\sigma}_{x}=\int_{-t / 2}^{t / 2} z \sigma_{x} d z, \quad \hat{\sigma}_{y}=\int_{-t / 2}^{t / 2} z \sigma_{y} d z, \quad \hat{\sigma}_{x y}=\int_{-t / 2}^{t / 2} z \sigma_{x y} d z \\
\hat{\gamma}_{x}=\int_{-t / 2}^{t / 2} \gamma_{x z} d z, \quad \hat{\gamma}_{y}=\int_{-t / 2}^{t / 2} \gamma_{y z} d z
\end{gathered}
$$

where $z$ has to be redefined as will be seen later.

According to the classification of the existing theories, the proposed scheme is a modification of the DBESL, where it is taken into account the evolution of the eccentricity of geometric and mechanical planes of a 

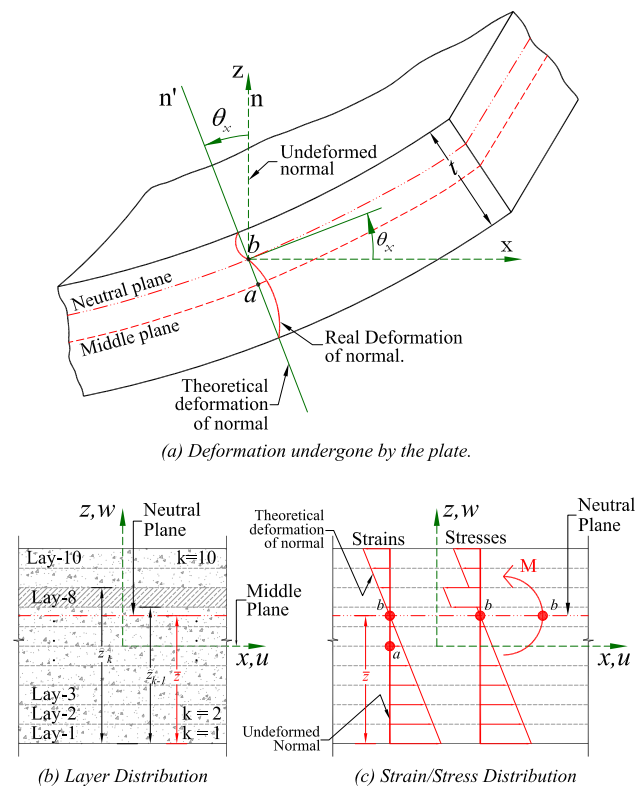

Fig. 3 Proposed deformation for a thin plate.

bi-dimensional element during the damage process, also, generalized stresses and strains have been referred to the mechanical plane, not to the geometrical plane as occurs in the classical DB-ESL formulation.

\subsection{FE implementation}

Although the proposed integration scheme is easily extensible to any bi-dimensional plate element, here the two-dimensional triangular FE developed by Batoz et al [8], depicted in figure 4 and commonly referred as the DKT element, has been taken as the starting point. The D.O.F. for element DKT have been presented in equation 1, and such element is based upon the assumption listed below.

1. Rotations vary quadratically over the element

$$
\theta_{x}=\sum_{i=1}^{6} N_{i} \theta_{x i} \quad ; \quad \theta_{y}=\sum_{i=1}^{6} N_{i} \theta_{y i}
$$
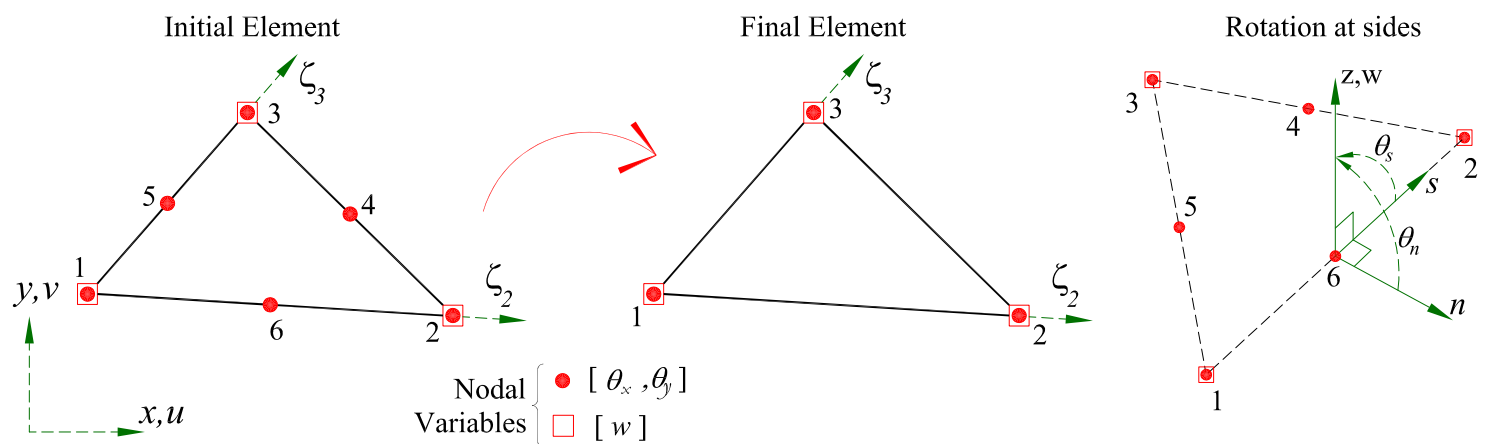

Fig. 4 Bi-dimensional triangular plate element, commonly called DKT element. 
where $\theta_{x}$ and $\theta_{y}$ are the nodal values at the corners and at the mid-nodes [fig.4], and $N_{i}(\xi, \eta)$ are shape functions.

$$
\begin{array}{ll}
N_{1}=2(1-\xi-\eta)\left(\frac{1}{2}-\xi-\eta\right) & N_{2}=\xi(2 \xi-1) \\
N_{3}=\eta(2 \eta-1) & N_{4}=4 \xi \eta \\
N_{5}=4 \eta(1-\xi-\eta) & N_{6}=4 \xi(1-\xi-\eta)
\end{array}
$$

2. The Kirchhoff hypothesis is imposed at corners (nodes 1,2,3)

$$
\theta_{x}+\frac{\partial w}{\partial x}=0 ; \theta_{y}+\frac{\partial w}{\partial y}=0
$$

and at the mid-nodes $(k=4,5,6)$

$$
\left(\theta_{s}\right)_{k}+\left(\frac{\partial w}{\partial s}\right)_{k}=0
$$

3. The variation of $w$ along the sides is cubic

$$
\left(\frac{\partial w}{\partial s}\right)_{k}=-\frac{3}{2 l_{i j}} w_{i}-\frac{1}{4}\left(\frac{\partial w}{\partial s}\right)_{i}+\frac{3}{2 l_{i j}} w_{j}-\frac{1}{4}\left(\frac{\partial w}{\partial s}\right)_{j}
$$

with $k=4,5,6$ denoting the mid-node of side $i j=23,31,12$ respectively, and $l_{i j}$ equal to the length of the side $i j$.

4. A linear variation of $\theta_{n}$ is imposed along sides

$$
\left(\theta_{n}\right)_{k}=\frac{1}{2}\left[\left(\theta_{n}\right)_{i}+\left(\theta_{n}\right)_{j}\right]
$$

The evaluation of the stiffness matrix for the DKT element follow the standard procedures of the finite element method, as depicted in equation 6.

$$
\mathbf{K}=\int_{A} \mathbf{B}^{\mathrm{T}} \mathbf{D}_{b} \mathbf{B} d A=2 A \int_{0}^{1} \int_{0}^{1-\zeta_{3}} \mathbf{B}^{\mathrm{T}} \mathbf{D}_{b} \mathbf{B} d \zeta_{2} d \zeta_{3}
$$

where $\mathbf{K}$ is the stiffness matrix, $A$ is the area of the FE, the deformation matrix $\mathbf{B}$ is defined as

$$
\mathbf{B}=\frac{1}{2 A}\left[\begin{array}{c}
y_{31} \mathbf{H}_{1}^{\mathrm{T}}+y_{12} \mathbf{H}_{3}^{\mathrm{T}} \\
-x_{31} \mathbf{H}_{2}^{\mathrm{T}}-x_{12} \mathbf{H}_{4}^{\mathrm{T}} \\
-x_{31} \mathbf{H}_{1}^{\mathrm{T}}-x_{12} \mathbf{H}_{3}^{\mathrm{T}}+y_{31} \mathbf{H}_{2}^{\mathrm{T}}+y_{12} \mathbf{H}_{4}^{\mathrm{T}}
\end{array}\right]
$$

being vectors $\mathbf{H}_{1}-\mathbf{H}_{4}$ functions of $\zeta_{2}$ and $\zeta_{3}$ defined in [8].

As will be seen later, the evaluation of the flexural stiffness for a plate element $\mathbf{D}_{b}$ is going to be the cornerstone of this work, let us first point out that the corresponding value for a continuum material, as the one depicted in figure 2 can be evaluated using equation 8 .

$$
\mathbf{D}_{b}=\int_{-\frac{t}{2}}^{+\frac{t}{2}} z^{2} \mathbf{D} d z
$$


The matrix $\mathbf{D}$ corresponds to the constitutive matrix of the material in plane stress. As can be seen in equation 9, $\mathbf{D}$ has not been integrated along the thickness of the plate.

$$
\mathbf{D}=\left[\begin{array}{ccc}
d_{11} & d_{12} & 0 \\
d_{21} & d_{22} & 0 \\
0 & 0 & d_{33}
\end{array}\right]
$$

However, for a laminated material formed by materials with different mechanical properties, the previous approach is limited, and it becomes necessary to express the flexural stiffness $\mathbf{D}_{b}$ in terms of the geometrical and mechanical properties of the $k_{t h}$ layer [11], leading to equation 10, which basically is a more general form of equation 8 , since $\mathbf{D}$ is no longer constant along the thickness, hence

$$
\mathbf{D}_{b}=\sum_{k=1}^{n} \frac{1}{3}\left(z_{k}^{3}-z_{k-1}^{3}\right) \mathbf{D}^{k}
$$

where $\mathbf{D}_{i j}$ has been defined in equation $9, k=1, \ldots, n$ is the current layer, and $n$ the total number of layers within the laminated material.

Figure 2.c depicts a typical distribution of stresses and strains within a laminated material, although properly speaking, it is not a laminated material at all, since it is composed by a one-single material. In any case, figure 2 is necessary as a reference to locate the layers, the middle plane and to state the position parameters $z_{k-1}$ and $z_{k}$ within a laminated material (fig. 2.b).

In order to perform the proposed integration scheme over the thickness, equation 10 has to be redefined, position parameters $z_{k}$ and $z_{k-1}$ have to be redefined also. For convenience, they are named $\hat{z}_{k}$ and $\hat{z}_{k-1}$ and referred to the bottom of the plate now, as shown in figure 3.b. This lead us to the cornerstone of the proposed scheme, which is the assessment of the neutral axis $\overline{\mathbf{Z}}$. To define $\overline{\mathbf{Z}}$, let us start defining the auxiliary matrix $\hat{\mathbf{D}}_{b}$.

$$
\hat{\mathbf{D}}_{b}=\int_{-\frac{t}{2}}^{+\frac{t}{2}} \bar{y}_{k} \mathbf{D}^{k} d z=\sum_{k=1}^{n} \bar{y}_{k} t_{k} \mathbf{D}^{k}
$$

in equation 11 , for convenience $\bar{y}_{k}=\left(\hat{z}_{k}+\hat{z}_{k-1}\right) / 2$, whereas $t_{k}=\hat{z}_{k}-\hat{z}_{k-1}$, this is, the thickness of the layer $k$, now it is possible to define $\overline{\mathbf{Z}}$ using equation 12 .

$$
\overline{\mathbf{Z}}=\left[\begin{array}{ccc}
\frac{\hat{\mathrm{D}}_{11}^{b}}{\mathrm{D}_{11}^{m}} & 0 & 0 \\
0 & \frac{\hat{\mathrm{D}}_{22}^{b}}{\mathrm{D}_{22}^{m}} & 0 \\
0 & 0 & \frac{\hat{\mathrm{D}}_{33}^{b}}{\mathrm{D}_{33}^{m}}
\end{array}\right]
$$

where the membrane stiffness $\mathbf{D}_{m}$ also has a role to play in the scheme and is defined as

$$
\mathbf{D}_{m}=\sum_{k=1}^{n}\left(\hat{z}_{k}-\hat{z}_{k-1}\right) \mathbf{D}^{k}
$$

Using equation 12, the bending stiffness tensor $\mathbf{D}_{b}$ from equation 10 is rewritten into equation 14 .

$$
\mathbf{D}_{b}=\sum_{k=1}^{n}\left[\frac{t_{k}^{3}}{12}+t_{k}\left(\hat{\mathbf{Z}}^{k}\right)^{2}\right] \mathbf{D}^{k}
$$


finally, $\hat{\mathbf{Z}}^{k}$ from equation 14 is defined in equation 15.

$$
\hat{\mathbf{Z}}^{k}=\bar{y}_{k} \mathbf{I}-\overline{\mathbf{Z}}
$$

Being I the identity matrix. With this new expression (eq. 14) the bending stiffness tensor is modified by non-linear effects, result of material failure, by two different means. One corresponds to the variation of the material stiffness matrix, which now has a lower stiffness as it is replaced by the secant tensor, and also by the position of the layer respect the new neutral axis of the laminate.

Shall be remarked that $\overline{\mathbf{Z}}$ will be evaluated at the beginning of a quasistatic loading process, and then in every iteration once damage has occur within the given FE. Also, has to be pointed out that this integration scheme is intended to be used with a secant tensor $\mathbf{D}^{\text {sec }}$ instead of $\mathbf{D}$ in equations 11 , and 14 , this in order to reproduce the change in the position of the mechanical plane using non-elastic constitutive equation.

Using equation 16 is now possible to evaluate the in-plane strains for each of the layers.

$$
\boldsymbol{\varepsilon}^{k}=\left\{\begin{array}{c}
\varepsilon_{x} \\
\varepsilon_{y} \\
\gamma_{x y}
\end{array}\right\}^{k}=-\hat{\mathbf{Z}}^{k}\left\{\begin{array}{c}
\frac{\partial \theta_{x}}{\partial x} \\
\frac{\partial \theta_{y}}{\partial y} \\
\frac{\partial \theta_{x}}{\partial y}+\frac{\partial \theta_{y}}{\partial x}
\end{array}\right\}
$$

the corresponding stresses can be evaluated using equation 17.

$$
\sigma^{k}=\mathbf{D}_{m}^{k} \varepsilon^{k}
$$

where $\boldsymbol{\sigma}^{k}=\left[\sigma_{x} \sigma_{y} \sigma_{x y}\right]^{\top}$. Finally, using $\hat{\mathbf{Z}}^{k}$ the integration through the thickness can be carried out using equation 18 .

$$
\hat{\boldsymbol{\sigma}}=\sum_{k=1}^{n} \hat{\mathbf{Z}}^{k} \boldsymbol{\sigma}^{k}
$$

where the vector of generalized stresses is defined as $\hat{\sigma}=\left[\hat{\sigma}_{x} \hat{\sigma}_{y} \hat{\sigma}_{x y}\right]^{\top}$. 


\section{Constitutive Formulation}

In this section a brief description of the expected bending degradation is carried out, focusing on the evaluation of the secant constitutive tensor $\mathbf{D}^{\text {sec }}$ which is required to reproduce the bending degradation that arises when some layers within a laminated plate are beyond the elastic threshold and others are not.

\subsection{Mechanical plane of a bending plate}

Let us start considering the example shown in figure 5 where the layer distribution of a plate under a monotonic bending stress is depicted. Layers of the laminated plate are formed by a homogeneous and isotropic material, in such a way mechanical plane lays in the geometrical plane. Let us also consider that the simple material forming the laminated plate is a concrete-like material, where there is a pronounced difference among tension and compression damage threshold.

As bending moment monotonic load starts being applied, the expected distribution of strains along the thickness would be the shown in figure 5.a, whereas stress distribution would be like the ones shown in figure 5.b. Both stresses and strains corresponding to figures 5.a and 5.b would be in the elastic range.

As bending moment increases, material non-linearity would be reached. The expected response of the laminate, in case of considering simple concrete, is such that only the layers under tension stress (since tension is by far the less resistant stress) reach the non-linear range, and due to this, layers subjected to compression forces undergo a gradual increase of stress. Consequently, strain level of a the composite material slightly beyond the damage threshold would be as shown in figure 5.c, also, from such figure notice that mechanical and geometrical axes no longer correspond. On the other hand, expected stress distribution would be presented in figure 5.d.

Finally, if it stills imposing a bending moment up to a fully damaged state in the plate, strain distribution would be very much alike at the ones presented in figure 5.e and the corresponding stress distribution shall be the one shown in figure 5.f. The movement of the mechanical plane, using the same definition of equation 2 , is found between the values:

$$
\hat{\mathbf{Z}}_{i i}^{k} \in\left[-\frac{t}{2},+\frac{t}{2}\right]
$$

being $i i$ the diagonal terms of $\hat{\mathbf{Z}}^{k}$ matrix. In order to get better accuracy to determine $\hat{\mathbf{Z}}^{k}$, becomes mandatory to perform a finer layer distribution in the following cases:

- At the farthest zones away from the geometric axis of the shell, since such layers will be subjected to the higher stresses while acting a bending stress.

- At zones where exists an abrupt change of stiffness, as is the case of the presence of steel reinforcement within the concrete, no matter their position within the overall thickness.

The main idea of such layer distribution is to endow the proposed integration scheme, with the capability of capturing the mechanical axis once the shell element has undergone a non-linear effect. The assumption is that, when the element damages, the mechanical axis moves away from its original position toward one end (according to the bending moment's direction), and the nearest to one end, the more damage state is able to represent.

\subsection{Isotropic Damage Model}

Continuum damage models (CDM) have been widely accepted as an alternative to deal with complex constitutive behaviour $[12,13,14,15]$. Among the different possibilities such a framework offers, the simplest is the one referring to isotropic damage models where the non-linear behavior is monitored through a single internal scalar variable called damage or degradation, $d$. The meaning of internal variable $d$ is the measurement of the loss of secant stiffness of the material, and it ranges from 0 for the undamaged material to 1 for the fully degraded one $(0 \leq d \leq 1)[16,17]$. The constitutive equation for an isotropic damage model has the form: 


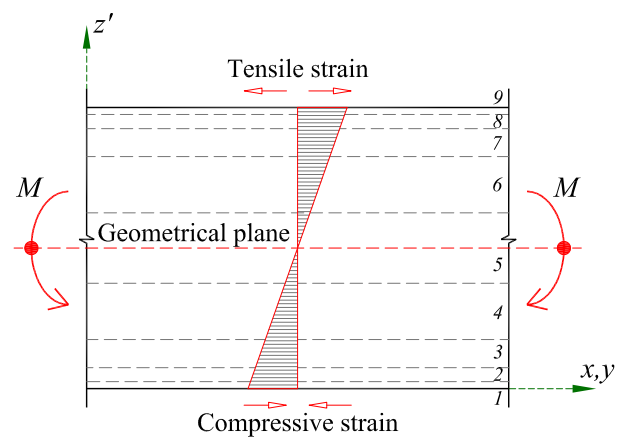

(a)

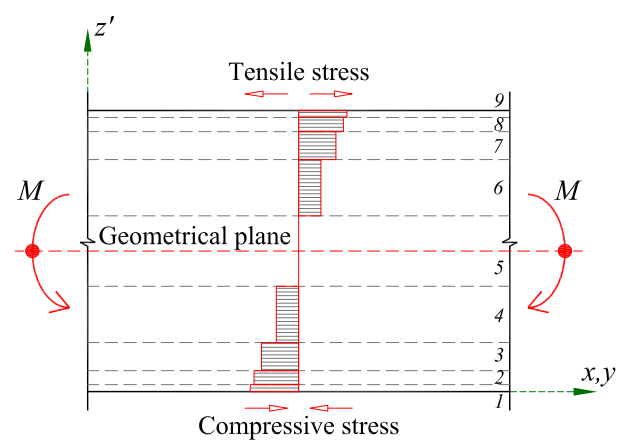

(b)

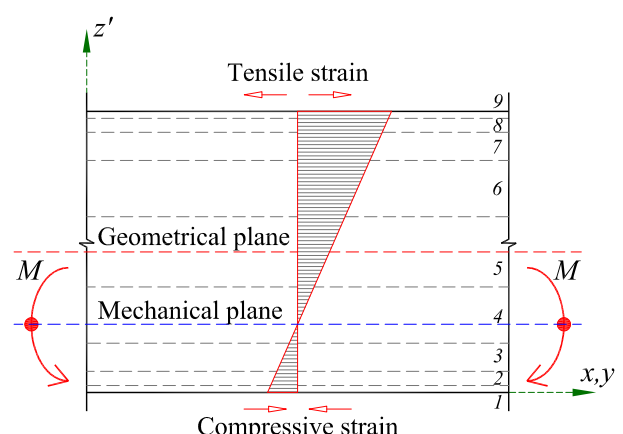

(c)

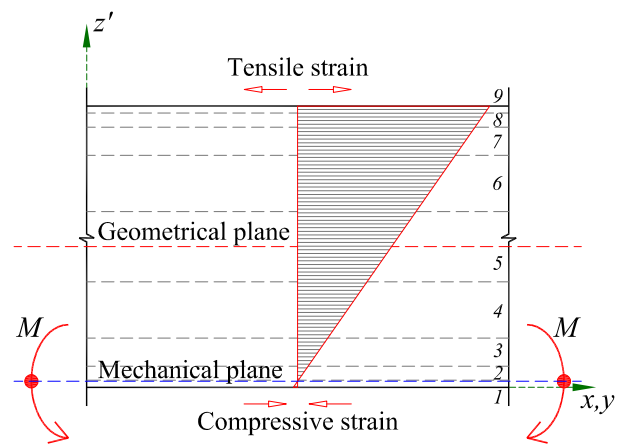

(e)

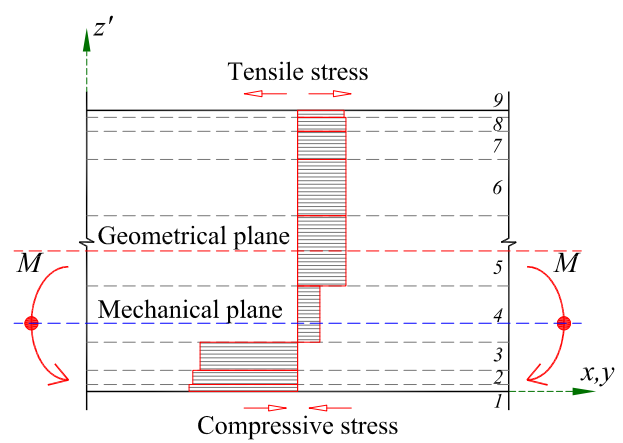

(d)

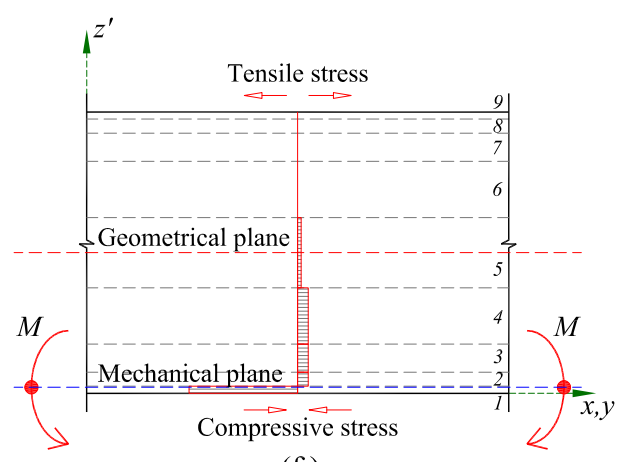

(f)

Fig. 5 Schematic representation of range to move of mechanical axis. 


$$
\boldsymbol{\sigma}=(1-d) \mathbf{D}: \boldsymbol{\varepsilon}
$$

and the secant stiffness $\mathbf{D}^{\text {sec }}$ is defined as

$$
\mathbf{D}^{s e c}=(1-d) \mathbf{D}
$$

Is important to remark that in the proposed scheme, during the degradation process, the gradual loss of bending stiffness will be evaluated using the secant constitutive tensor of each layer. An overview of the components conforming the model proposed by Oliver et al [16] is summarized in next paragraph.

a. a suitable norm, $\tau$, used to compare different states of the deformation making possible to define concepts such as loading, unloading and reloading.

b. a damage criterion, $\mathbf{F}(\tau, r) \leq 0$, formulated in the strain or the undamaged stress space. The simplest form of this criterion is:

$$
\mathbf{F}(\tau, r)=\mathbf{G}(\tau)-\mathbf{G}(r) \leq 0
$$

where $\tau$ is the norm described in $(a), r$ is the damage threshold, and $\mathbf{G}(\bullet)$ is a suitable monotonic scalar function. Using equation 22, now damage is numerically defined to occur when $\mathbf{G}(\tau)>\mathbf{G}(r)$.

c. evolution laws for the damage threshold and the damage variable. Such laws are given by the rate expressions:

$$
\begin{aligned}
& \dot{r}=\dot{\mu} \\
& \dot{d}=\dot{\mu} \frac{\partial \mathbf{F}(\tau, r)}{\partial \tau}=\dot{\mu} \frac{d \mathbf{G}(\tau)}{d \tau}
\end{aligned}
$$

where $\dot{\mu}$ is the damage consistency parameter used to define loading/unloading conditions according to the Kuhn-Tucker relations.

$$
\dot{\mu} \geq 0 \quad ; \quad \mathbf{F}(\tau, r) \leq 0 \quad ; \quad \dot{\mu} \mathbf{F}(\tau, r)=0
$$

The norm $\mathbf{G}(\tau)$ together with the damage criterion play the important role of defining the yield surface, this is when $\mathbf{G}(\tau)-\mathbf{G}(r)=0$, this is particularly important, since most of the common yield surface can be reproduced using an isotropic damage model, like the ones proposed by are Tresca and Mohr-Coulomb, or the modification to the Mohr-Coulomb yield surface proposed by Oller [18] which are suitable to model concrete-like materials. Ductile materials such as metals, on the other hand, can also be modeled using a von Mises yield surface.

\subsection{Secant tensor for elasto-plastic materials}

Including the evolution of permanent deformations or plastic deformations in a CDM allows to represent the behavior of ductile materials with an elasto-plastic behavior.

Although in this work the proposed scheme only focuses on an isotropic CDM, the concepts presented here can also be extended to plastic damage models, like the one proposed by Oller in [18] where it is used a normalized internal variable to represent the plastic damage $\kappa^{p}$. Range of values for $\kappa^{p}$ are $0 \leq \kappa^{p} \leq 1$, such that if $\kappa^{p}=0$ there is no plastic damage, and $\kappa^{p}=1$ defines the total damage of a solid.

There are also other CDM which include the presence of permanent deformations on the mechanical behavior of geomaterials $[19,20,21,22]$ especially in concrete subjected to compression. Such schemes make use of internal variables to represented damage and permanent plastic deformation. 
The idea of using one single damage variable in a general form has been explored by Paredes [23] to be applied in the assess of the natural frequency of vibration of structures. Paredes considers the different existing possibilities to define the damage index $d$, and proposes the index $d^{\text {eqv }}$ be a function of the yield surface $f(\sigma)$ defined as:

$$
d^{e q v}=1-\frac{f\left(\sigma^{c}\right)}{f(\sigma)}
$$

hence, it is now possible to use the damage index $d^{\text {eqv }}$ in equation 21 to obtain an equivalent secant tensor. In equation $25 f(\sigma)$ is evaluated with the current tensor of stress, whereas $f\left(\sigma^{c}\right)$ is evaluated with the tensor of effective stresses that define the damage threshold of the material which is defined as

$$
\sigma^{c}=\left[\begin{array}{lll}
f^{*} & 0 & 0 \\
0 & 0 & 0 \\
0 & 0 & 0
\end{array}\right]
$$

where $f^{*}$ is nominal threshold of the material's resistance. For the case of concrete, such threshold is defined by the resistance to compression, whereas for ductile materials such as steel, it is defined by the yield stress.

Finally, the use of equation 25 takes to the modification of equation 21 resulting in equation 27.

$$
\mathbf{D}^{s e c}=\left(1-d^{e q v}\right) \mathbf{D}
$$

\subsection{Two-scalar damage models}

Other damage model highly extended to model the opening and closing of cracks due cyclic loads in geomaterials is the one proposed by Faria et al in [24], here two scalar damage variables $d^{+}$and $d^{-}$are introduced as internal variables, so it is possible to distinguish among the damage produced by tensile stresses (cracking) and the damage produced due compressive stresses (crushing). The nature of the scheme proposed by Faria et al makes it more feasible for seismic analysis purposes.

Using the definition of the elastic secant constitutive tensor, the secant constitutive tensor can be evaluated for this scheme as [24]:

$$
\mathbf{D}^{s e c}=\frac{\partial \boldsymbol{\sigma}}{\partial \boldsymbol{\varepsilon}}=\left(1-d^{+}\right) \frac{\partial^{2} \Psi_{0}^{+}}{\partial \boldsymbol{\varepsilon}^{e} \otimes \partial \boldsymbol{\varepsilon}^{e}}+\left(1-d^{-}\right) \frac{\partial^{2} \Psi_{0}^{-}}{\partial \boldsymbol{\varepsilon}^{e} \otimes \partial \boldsymbol{\varepsilon}^{e}}
$$

The use of two internal variables to reproduce the damage is an advantage in terms of the constitutive analysis. On the other hand, it becomes cumbersome while trying to establish one single parameter that define the degradation of the material. This can only be achieved with an equivalent damage index [25] (eq. 27).

\subsection{Secant tensor for composite materials}

The use of mixing theories allows the numerical simulation of composite materials formed by $n$ simple materials, being also possible to represent each of them with their own constitutive model. This is because mixing theories function as a manager of constitutive models whose main function is to preserve the compatibility of deformations, the equilibrium of internal forces, and to take into account the corresponding stiffness of each component.

Classical mixing theory (CMT), whose simpler expression is the rule of mixtures (ROM), was first studied in 1960 [26] establishing the basis for subsequent developments. CMT takes into account the volume fraction of the components but not its morphological distribution, since it assumes all component materials experiment a pure parallel behavior (same strain in all directions). To overcome this strong limitation Rastellini et al [27] developed a serial/parallel mixing theory (SP ROM) assuming that components behave as a parallel material in the fiber alignment direction and as serial material in the orthogonal direction. SP ROM will only be mentioned here, and reader may consult [27, 28] for a full reference for the basic 
notations, definition and computational implementation. Also reader may consult references [29, 30] where SP ROM formulation has been used to characterize reinforced concrete structures.

During a loading process, if any of the component materials reach the non-linear range, the constitutive tensor of such material will change, and consequently, so the constitutive tensor of the composite material. Hence, to take into account such change, the use of equations 29, 30 and 31 depicts the way the secant tensor has to be evaluated using a SP ROM scheme.

$$
\begin{aligned}
& \mathbf{D}_{p p}^{s e c}=\left({ }^{f} k^{f} \mathbf{D}_{p p}^{s e c}+{ }^{m} k^{m} \mathbf{D}_{p p}^{s e c}\right)+{ }^{m} k^{f} k\left({ }^{f} \mathbf{D}_{p s}^{s e c}-{ }^{m} \mathbf{D}_{p s}^{s e c}\right): \mathbf{A}:\left({ }^{m} \mathbf{D}_{s p}^{s e c}-{ }^{f} \mathbf{D}_{s p}^{s e c}\right) \\
& \mathbf{D}_{p s}^{s e c}=\left({ }^{f} k^{f} \mathbf{D}_{p s}^{s e c}: \mathbf{A}:{ }^{m} \mathbf{D}_{s s}^{s e c}+{ }^{m} k^{m} \mathbf{D}_{p s}^{s e c}: \mathbf{A}:{ }^{f} \mathbf{D}_{s s}^{s e c}\right) \\
& \mathbf{D}_{s p}^{s e c}=\left({ }^{m} k^{f} \mathbf{D}_{s s}^{s e c}: \mathbf{A}:{ }^{m} \mathbf{D}_{s p}^{s e c}+{ }^{f} k^{m} \mathbf{D}_{s s}^{s e c}: \mathbf{A}:{ }^{f} \mathbf{D}_{s p}^{s e c}\right) \\
& \mathbf{D}_{s s}^{s e c}=\frac{1}{2}\left({ }^{m} \mathbf{D}_{s s}^{s e c}: \mathbf{A}:{ }^{f} \mathbf{D}_{s s}^{s e c}+{ }^{f} \mathbf{D}_{s s}^{s e c}: \mathbf{A}:{ }^{m} \mathbf{D}_{s s}^{s e c}\right)
\end{aligned}
$$

where

$$
\mathbf{A}=\left({ }^{m} k^{f} \mathbf{D}_{s s}^{s e c}+{ }^{f} k^{m} \mathbf{D}_{s s}^{s e c}\right)^{-1}
$$

and, $\mathbf{D}_{p p}^{s e c}$ is the component in parallel direction of the secant tensor, $\mathbf{D}_{s s}^{s e c}$ is the component in serial direction of the secant tensor, and $\mathbf{D}_{p s}^{s e c}$ and $\mathbf{D}_{s p}^{s e c}$ are the components in serial and parallel direction. Finally, the secant tensor for a composite material using a SP ROM is:

$$
\mathbf{D}^{s e c}=\left[\begin{array}{ll}
\mathbf{D}_{p p}^{s e c} & \mathbf{D}_{p s}^{s e c} \\
\mathbf{D}_{s p}^{s e c} & \mathbf{D}_{s s}^{s e c}
\end{array}\right]
$$

Similarly, using the CMT, the secant constitutive tensor of a composite material is equal to

$$
\mathbf{D}^{s e c}=\sum_{i=1}^{n}{ }^{i} k^{i} \mathbf{D}^{s e c}
$$

where ${ }^{i} k$ and ${ }^{i} \mathbf{D}^{s e c}$ are respectively the volumetric participation and the secant constitutive tensor of material $i$. In both cases, using either CMT or SP ROM $\mathbf{D}^{s e c}$ of the constituent materials have to be obtained using either equation 21 or equation 27.

\subsection{Mesh objectivity of FE formulation response with strain softening}

In continuum mechanics, it is known that the inclusion of strain-softening leads to the increment of strains in narrow strips. This phenomenon is known as strain localization. Two major approaches may be distinguished for analyzing crack propagation, namely: Discontinuous (or Discrete) Crack Approach (DCA) and the Smeared Crack Approach (SCA).

The purpose of this section is only to point out in a briefly manner the strain-softening phenomena and to describe the SCA, since is the approach used in this work. Reader may abound in the subject by consulting reference [31] where a comprehensive review of the formulations used over the last 40 years for the solution of problems involving tensile cracking, with both the DCA and the SCA has been carried out. Some brief remarks on the SCA are listed below.

- The simplicity of this approach. SCA can be readily implemented in any non-linear FE code, by simply writing a routine for a new material constitutive model.

- Re-meshing is unnecessary. Since the cracking material is assumed to remain continuum and material properties (stiffness and strength) are modified to account for the effect of cracking.

- Mesh dependency. A drawback is that the total energy dissipated in the cracking process is proportional to the size of the element. 


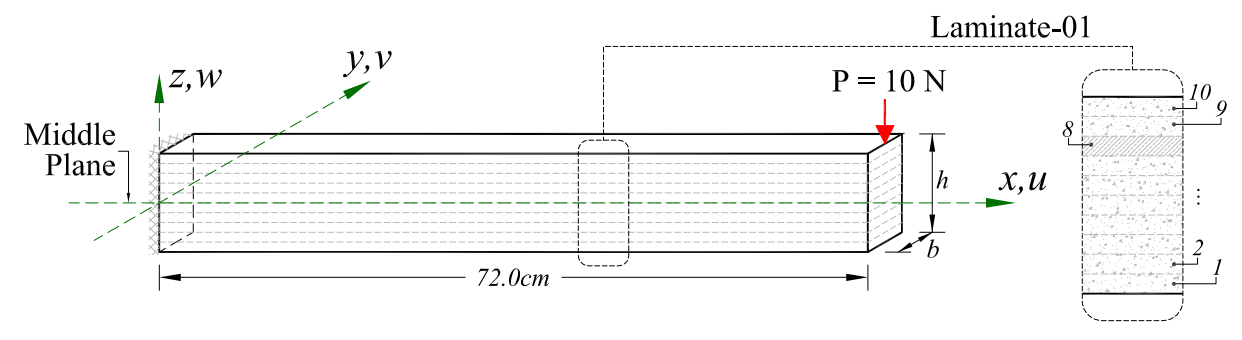

Fig. 6 Fixed beam subjected to flexion.

The requirement that the result from the numerical modelling should be independent of the mesh choice is named mesh objectivity. Several studies have been done to provide objectivity on the results independently of the size of the FE mesh (Oliver [32], Oller [33], Oliver et al [34], Cervera and Chiumenti [35] Lubliner et al [20], Martinez et al [36, 37], Paredes et al [25], among others).

\section{Practical Examples}

\subsection{Linear clamped beam}

The first example selected to demonstrate the efficiency of the proposed scheme is shown in figure 6. It is a beam fixed at one end with the other end free where a vertical load $P=10 \mathrm{~N}$ is applied. The total length of the beam is $L=0.72 \mathrm{~m}$, whereas the dimensions of the cross section are $b=0.04 \mathrm{~m}$ and $h=0.10 \mathrm{~m}$. The layer distribution of the laminated material is also depicted in figure 6 , the thickness of all layers are $t_{k}=0.01 \mathrm{~m}$, all except layer 08 are formed only by a concrete matrix. Layer 8 on the other hand, is a composite material formed by a volumetric participation of $90 \%$ of concrete which is reinforced with $10 \%$ of steel fibre. Composite material for layer 8 will be modeled using the SP ROM [28]. Both concrete and steel have been modeled using a elastic constitutive equation whose mechanical properties are $E_{c}=3.5 e 7 \mathrm{~N} / \mathrm{m}^{2}, v_{c}=0.20, E_{s}=2.1 \mathrm{e} 11 \mathrm{~N} / \mathrm{m}^{2}$ and $v_{s}=0.0$, where subindexes $c$ and $s$ stands for concrete and steel respectively.

Considering only $x$ direction in equations $11,12,14$, and 15 , then

$$
\begin{aligned}
& \hat{\mathrm{D}}_{1,1}^{b}=1.592 \times 10^{7} \mathrm{~N} \\
& \mathrm{D}_{1,1}^{m}=2.13 \times 10^{8} \mathrm{~N} / \mathrm{m} \\
& \bar{Z}_{1,1}=7.46 \times 10^{-2} \mathrm{~m} \\
& \mathrm{D}_{1,1}^{b}=5.75 \times 10^{-6} \mathrm{~m}^{4}
\end{aligned}
$$

also, using the analogy with the beam of Euler-Bernoulli with $E I=\mathrm{D}_{1,1}^{b}$, leads to state that

$$
\delta_{a}=\frac{P L^{3}}{3 \mathrm{D}_{1,1}^{b}}=-4.56 \times 10^{-3} \mathrm{~m} \quad ; \quad \theta_{a}=\frac{P L^{2}}{2 \mathrm{D}_{1,1}^{b}}=9.50 \times 10^{-3} \mathrm{rad}
$$

where $\delta_{a}$ and $\theta_{a}$ are the displacement and the rotation at point $a$ respectively (fig. 7).

The results shown in equation 34 will be used as the basis for the upcoming comparisons with two modelling strategies. The first strategy consist in a mesh with 365 nodes and 576 two-dimensional triangular FE combined with the proposed scheme, that for referencing purposes it will be named Sh01 from now on. The second modelling strategy consists in 2880 elements and 14,837 nodes formed by 20-node Lagrangian FEs, named Hex01 from now on.

The layer distribution for the laminated composite material depicted in figure 6 has been selected in order to make comparison in terms of vertical displacement, but especially, in terms of horizontal displacements among both models. 


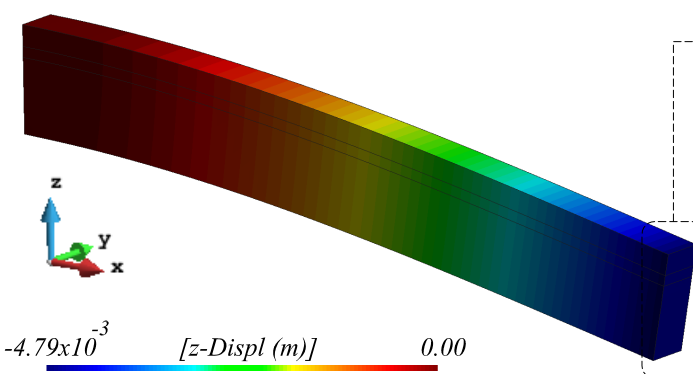

(a) Deformed mesh - Three-dimensional FE.

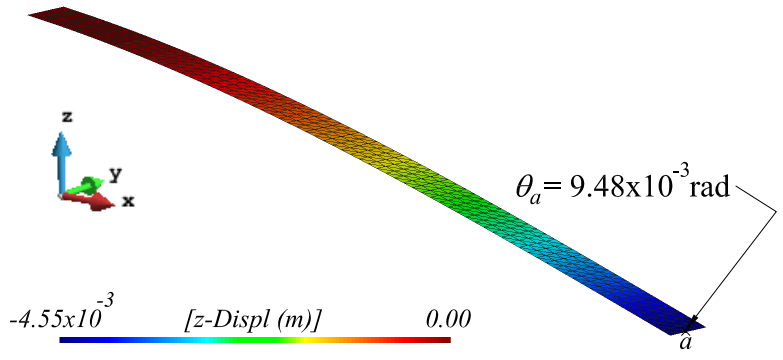

(b) Deformed mesh-Proposed scheme.

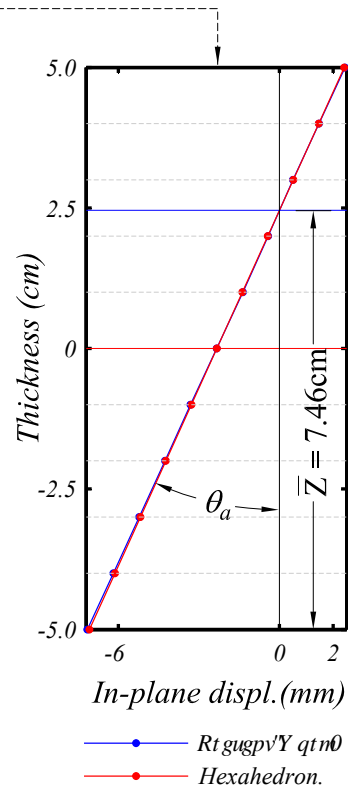

(c) Rotation comparison

Fig. 7 Vertical and in-plane displacement comparison.

The first point of comparison of the two models are the displacements shown in figure 7 . The maximum value obtained for the $z$ displacement for model Hex01 is $\delta_{H e x 01}=-4.79 \times 10^{-3} m$ (figure 7.a), while the value obtained with the proposed scheme is $\delta_{S h 01}=-4.55 \times 10^{-3} \mathrm{~m}$ (figure 7.b), the latter having better approximation compared with the value shown in equation 34 .

On the other hand, figure 7.c is used in order to show a comparison among the $x$ displacements of both models. Also in figure 7.c is depicted the value of $\bar{Z}_{1,1}$ obtained in eq. 33, which according to the proposed scheme, will be the reference plane to evaluate the in-plane displacements, and consequently the in-plane strains and stresses for a plate element. As can be seen from such figure, the relationship among the in-plane displacements for both models is exact. Values of the in-plane $x$ displacements for model Sh01 have been evaluated using the obtained value for the rotation $\theta_{S h 01}=9.48 \times 10^{-3} \mathrm{rad}$, whereas values for model Hex01 are the global displacements in $x$ direction.

Also, a comparison in terms of strains and stresses can be performed more accurately if layer 08 from model Sh01 is split into 8 layers, which leads to a total of 17 layers for model Sh01.

Comparison of the stresses undergone by steel fibres are presented in figure 8. Figure 8.a corresponds to obtained results for model Hex01. In such figure it is also possible to appreciate the change in the stress (compressive and tensile) within the same FE. This effect has been possible to be captured due to the type of FE used, since 4-node and 8-node three-dimensional elements are not able to cast reliable results under bending conditions, such FE have not been considered in this work. Hence, for bending cases, it is highly recommended a quadratic approximation or higher [7], which makes computationally expensive the used strategy for model Hex01, and naturally arises the reason of using the proposed scheme.

Figure 8.b, on the other hand, shows the stresses at the top of the reinforced layer for model Sh01, as can be seen, the maximum tensile stress undergone by such layer is $\sigma_{x x}=2.710 \times 10^{7} \mathrm{~N} / \mathrm{m}^{2}$, which matches the obtained for model Hex01 being $\sigma_{x x}=2.757 \times 107 \mathrm{~N} / \mathrm{m}^{2}$. In both cases, such values are obtained in the fixed end of the model. An excellent match among models is also achieved at the bottom part of the reinforced layer, which for model Sh01 is depicted in figure 8.c. Figure 8.c reports the minimum undergone stress $\sigma_{x x}=-2.228 \times 10^{7} \mathrm{~N} / \mathrm{m}^{2}$, whereas for model Hex01 $\sigma_{x x}=-2.204 \times 10^{7} \mathrm{~N} / \mathrm{m}^{2}$. 


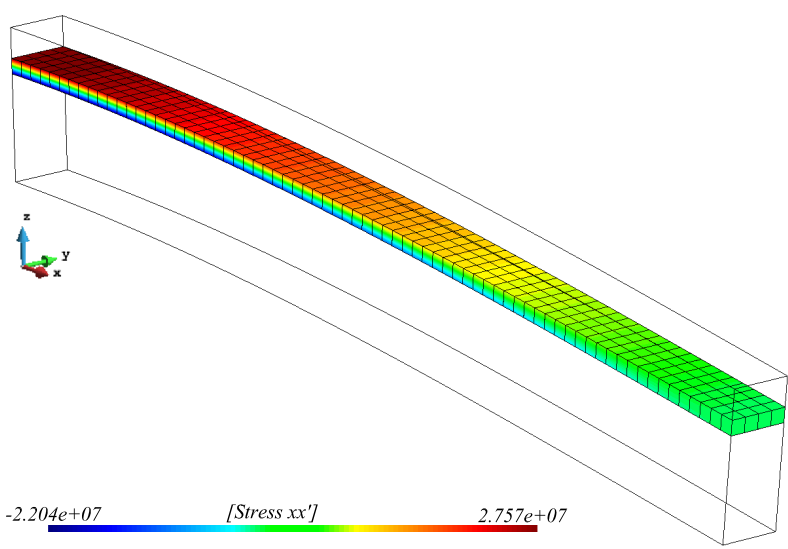

(a) Stresses tree-dimensional FE model.

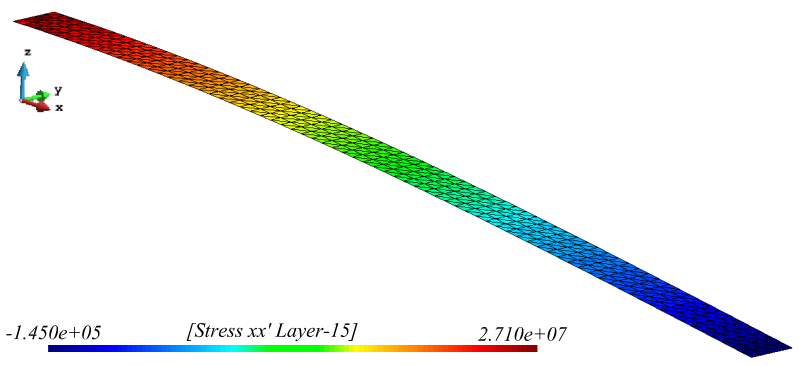

(b) Stresses bi-dimensional FE model.

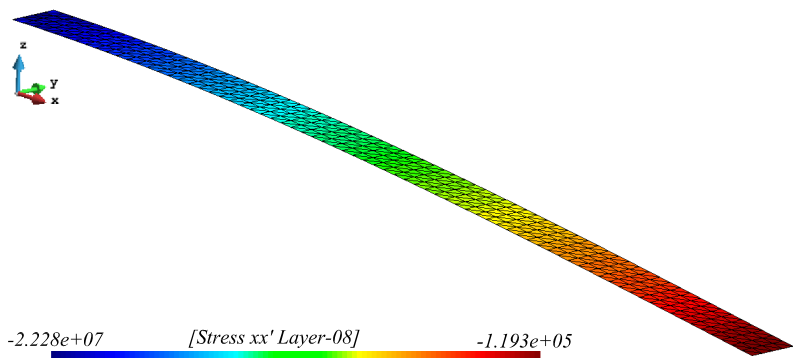

(c) Stresses bi-dimensional FE model.

Fig. 8 Stress comparison of steel reinforcement among models Hex01 and Sh01. 


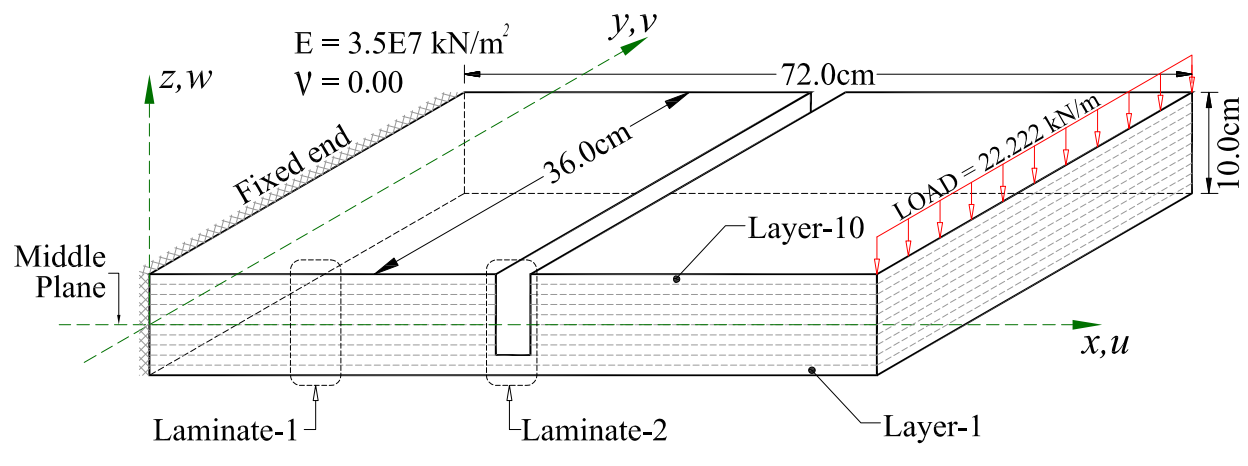

Fig. 9 Geometry and boundary conditions for a fixed plate.

\subsection{Linear clamped plate with a notch}

The proposed scheme is also efficient in cases where there are large discontinuities between the position of the mechanical plane of adjacent FE's. To demonstrate this fact, has been carried out the study shown in figure 9, where is depicted the geometry of a plate fixed in one of their edges and containing two different laminated materials. The layer distribution of both laminated materials is also depicted in figure 9 , as can be seen there, the basic difference among them is the notch imposed in laminated material 02 . Figure 9 also presents the loading conditions, mechanical properties of the material, and the geometry of the proposed model.

The analysis has been carried out using two meshes, the first one composed by 20-node Lagrangian three-dimensional element, and the second one with triangular DKT element with the proposed scheme. The obtained results are presented in figure 10, where at the top is presented the deformed mesh for both models, and at the bottom it is presented a comparison of the vertical displacement.

\subsection{Non-linear clamped plate}

Let us consider the example shown in figure 11, where a model subjected to bending moment is depicted. Two cases are considered, a shell element with the proposed integration scheme, and a conventional hexahedron element. In both cases, boundary conditions will be the same, namely, one end fixed, and in the other end, a vertical displacement monotonically increased will be imposed. Dimensions of the model are $72 \times 36 \times 10 \mathrm{~cm}$. For the shell model, it has been used a mesh with a total of 144 triangular elements with a single integration point, on the other hand, the model with hexahedron elements has been performed using a mesh with 720 finite elements with 8 integration points each.

Material properties of the plate are: Young's modulus $E_{x x}=E_{y y}=3.5 E 4 M P a$, Poisson's ratio $v=0.2$, internal friction angle $\phi=30^{\circ}$, compression strength $\sigma_{y c}=20 M P a$, tensile strength $\sigma_{y t}=2.0 M P a$, fracture energy $G_{f}=0.25 \mathrm{kN} / \mathrm{m}$ and compression energy $G_{c}=26.0 \mathrm{kN} / \mathrm{m}$. In order to stay away of the definition and evaluation of the fracture length, since for both bi-dimensional and three-dimensional elements different theory may apply, fracture length will be fixed in both examples to $l_{c h}=h_{g p}=3 \mathrm{~cm}$, where $h_{g p}$ is the length of a Gauss point in the load direction of a hexahedron element. Finally, to assess the secant tensor of each layer has been used equation 21 corresponding to an isotropic damage model.

Figure 12 depicts the evolution on the non-linear force-displacement response of both models. It is easy to notice the excellent agreement achieved between the two models, although the layered shell element slightly underestimated the response. Also it is easy to notice that bending moment at the support is equal to $M_{a}=P L$. Values corresponding to imposed displacements $\delta_{1}, \delta_{2}$ and $\delta_{3}$ are used as a reference to the information output for a random F.E (figures 13 and 14).

Once both analysis have been performed, the first comparison point is the deformation undergone by both models, such comparison is shown in figure 13, as expected, damage takes place in the nearest elements to the fixed end in both models. This seems obvious, since such elements are subjected to the highest levels 

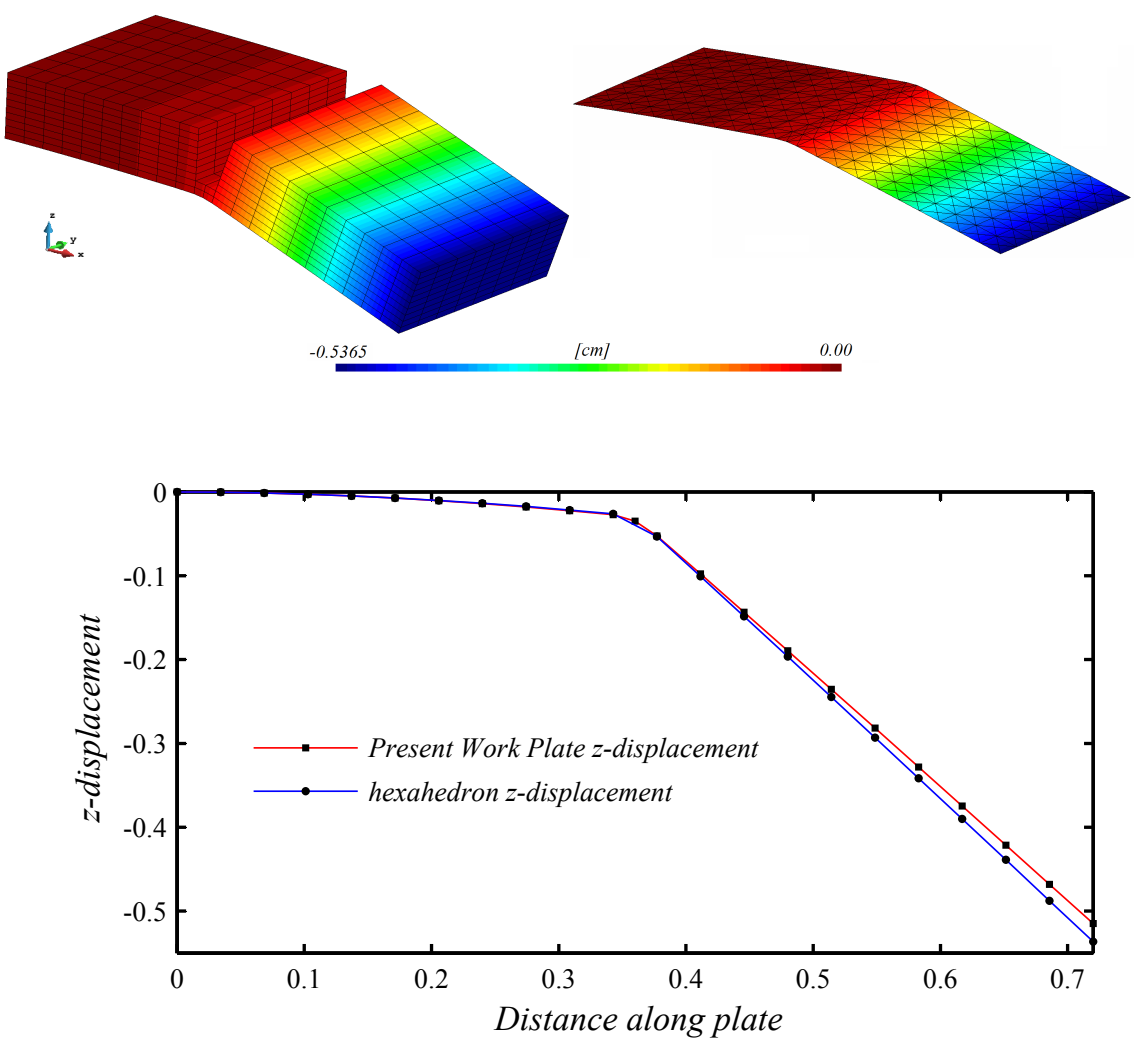

Fig. 10 Obtained results for a fixed plate.

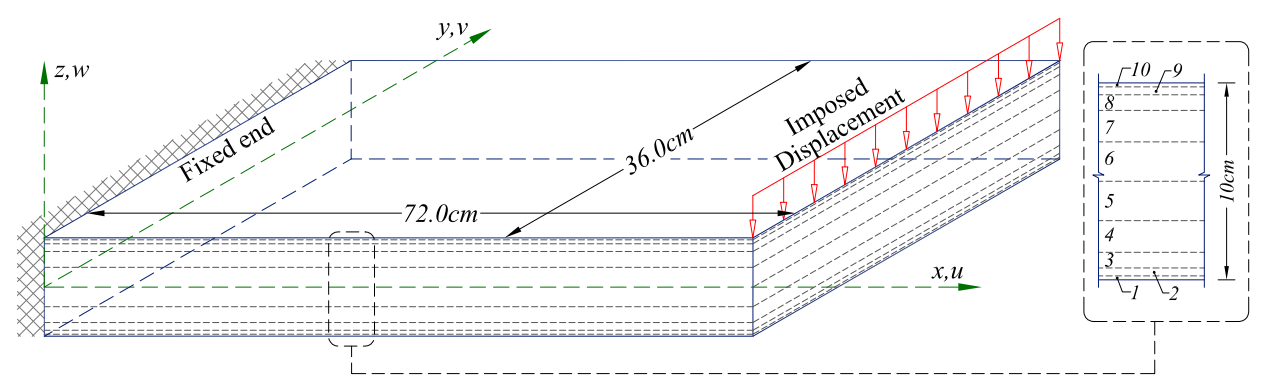

Fig. 11 Schematic representation of bending loaded plate. 


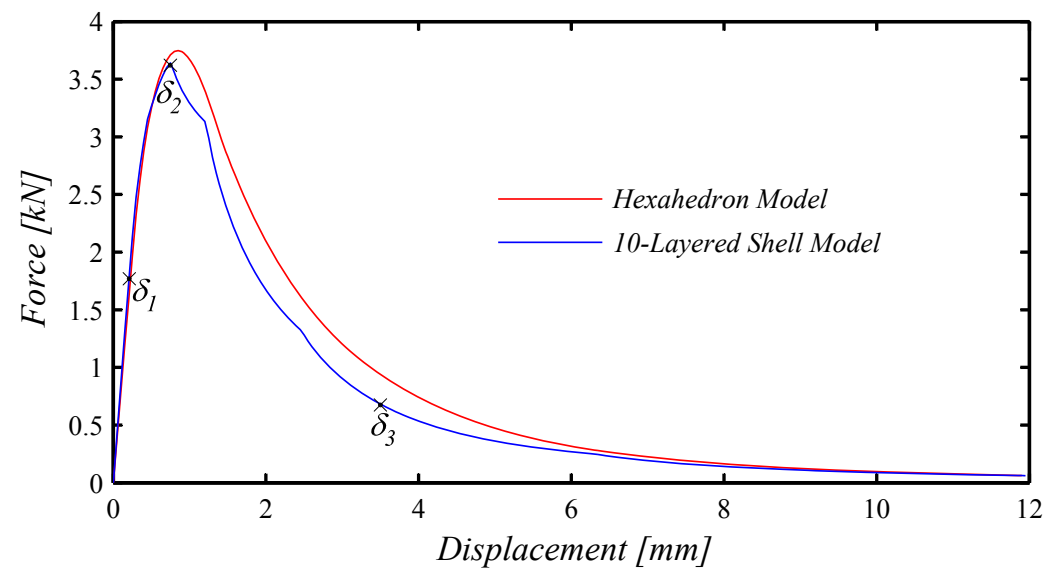

Fig. 12 Non-linear FEM response of a vertically loaded plate using $l_{c h}=3 \mathrm{~cm}$.
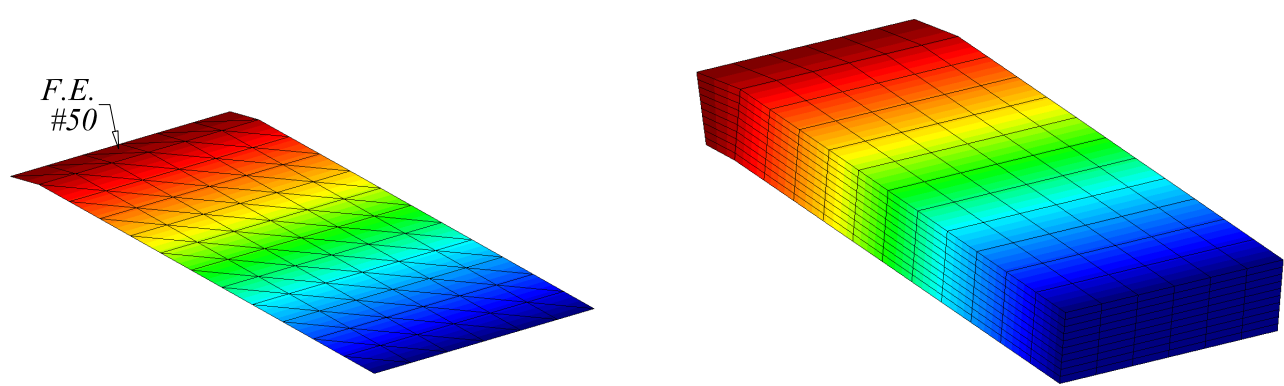

Fig. 13 Final deformation of vertically loaded models. 
of tensile stresses at theirs top layers. In both cases, damage starts at the top most layers/elements, then it propagates to the inner layers/elements.

In figures $14,(a),(b)$ and $(c)$ four graphics are shown, from left to right they correspond to: damage variable of the layer, strain distribution along thickness's element, stress distribution along thickness and generalized stress $\hat{\sigma}_{X}$. Such figures show the stresses and strains undergone by FE 50 (Fig. 13) at three representative loading stages during the loading process.

First loading stage (fig. 14.a) correspond to an initial state of stresses at the end of the elastic range, singe a slight damage begins at the top of the element. As seen, mechanical plane (blue horizontal line) matches the geometrical plane (red horizontal line). Strains are depicted as a straight vertical line due to the scale used to show the results (the same scale has been kept for the three figures), although such distributions shall look very much alike the one shown in figure 13.a. On the other hand, stress distribution remains symmetric in respect of geometrical plane.

The second loading stage is presented in figure 14.b, corresponds to the maximum bending stress in the shell $\left(M_{\max }=7.774 \mathrm{kN} \cdot \mathrm{m} / \mathrm{m}\right)$, and is also the point where damage along the layers of the element becomes more visible, since mechanical (blue) and geometrical plane (ref) no longer correspond.

Finally, loading stage shown in figure fig. 14.c corresponds to a high damaged state (although not fully damaged) where layers at the top of the element are fully damaged and a huge change in the position of the mechanical plane has occur.

Additionally, in order to point out the biggest advantage of using this scheme, is proposed a different model using the same geometry and boundary conditions shown in figure 11 with a slight change in the layer distributions, however, the total thickness remains the same. Such change is due to the addition of steel reinforcement fibers to enhance the bending resistance of the plate.

The layer provided with steel reinforcement is near the top of the laminate, its thickness is $T h_{i}=3 \mathrm{~mm}$, and has a volumetric participation of fiber equal to 5\% embedded in a matrix of concrete. Mechanical properties used for steel are: $E_{x x}=2.1 E 5 M P a ; E_{y y}=3.5 E 4 M P a$, Poisson's ratio $v=0$, compression strength $\sigma_{y c}=412 \mathrm{MPa}$, tensile strength $\sigma_{y t}=412 \mathrm{MPa}$, fracture energy $G_{f}=20.0 \mathrm{kPa} \cdot \mathrm{m}$ and compression energy $G_{c}=20.0 \mathrm{kPa} \cdot \mathrm{m}$. Constitutive model adopted for mechanical behaviour of steel is the one proposed by Oller in [18], where plastic internal variable $\kappa_{p}$ takes the place of $d$ in equation 21. To numerically reproduce the composite behavior, has been used the SP ROM [28, 38], and has been split into 6 layers to get a total amount of 15 layers within the laminated material.

The reason of using an non-symmetric arrangement of the layers with respect to the geometric plane, is to show that, if in the linear range there is a difference between the position of the geometric plane and the mechanical plane, such difference increases once the a monotonically increased displacement is applied.

Such effect can be appreciated in the obtained results once the analysis has been performed, a resume of such results is presented in figure 15. Figure 15.a shows a comparison among the results obtained for non-reinforced plate (previous example) and for a reinforced plate. There is a significant improvement in the bending strength even with a small amount of steel reinforcement, in addition, there is no longer a brittle failure, but degradation is presented in a more gradual manner.

The graph of figure 15.a also displays displacements $\delta_{1}, \delta_{2}, \delta_{3}$ and $\delta_{4}$ used as a reference for the output results for F.E. 50 shown in figure 13, whose stresses and strains undergone are presented from figure 15.b to 15.e. For display purposes different horizontal scales has been used in such figures.

The stresses and strains of studied FE corresponding to displacement $\delta_{1}=1.25 \mathrm{~mm}$ are depicted in figure 15.b. As can be seen, although at such loading step the element is in a linear range, already exists a difference between mechanical (blue line) and geometrical plane (red line). On the other hand, the maximum bending strength of the model is achieved when $\delta_{2}=2.30 \mathrm{~mm}$ (figure 15.c). From figure 15.b to $15 . \mathrm{d}$ it can be noticed that there is no significant difference on the stresses or the strains undergone by the element at those loading steps, neither there is a significant difference in the location of the mechanical axis. Figure 15.e, on the other hand, depicts a significant change in the stresses accompanied with slight change of strains. This is due to the degradation in the lower layers of the laminated material, this has caused a change in the location of the mechanical plane, now it is somewhere inside the reinforced layers, and some of them are under compressive forces.

The main advantage of the proposed scheme is to accurately reproduce the bending behavior of structures 

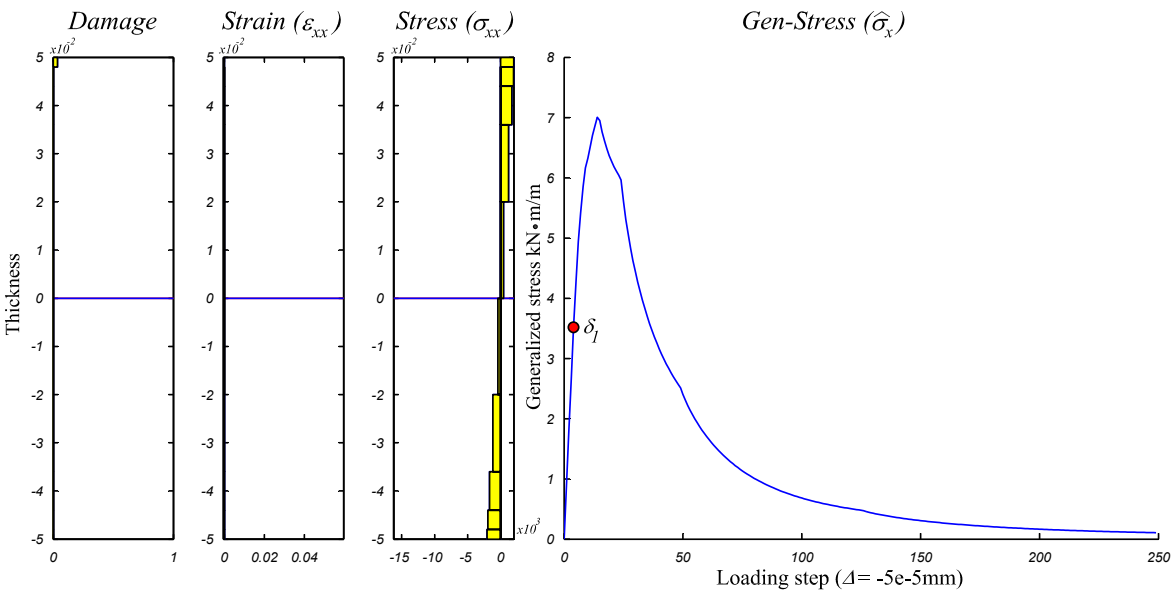

(a) Step 4, $\delta_{1}=0.20 \mathrm{~mm}$
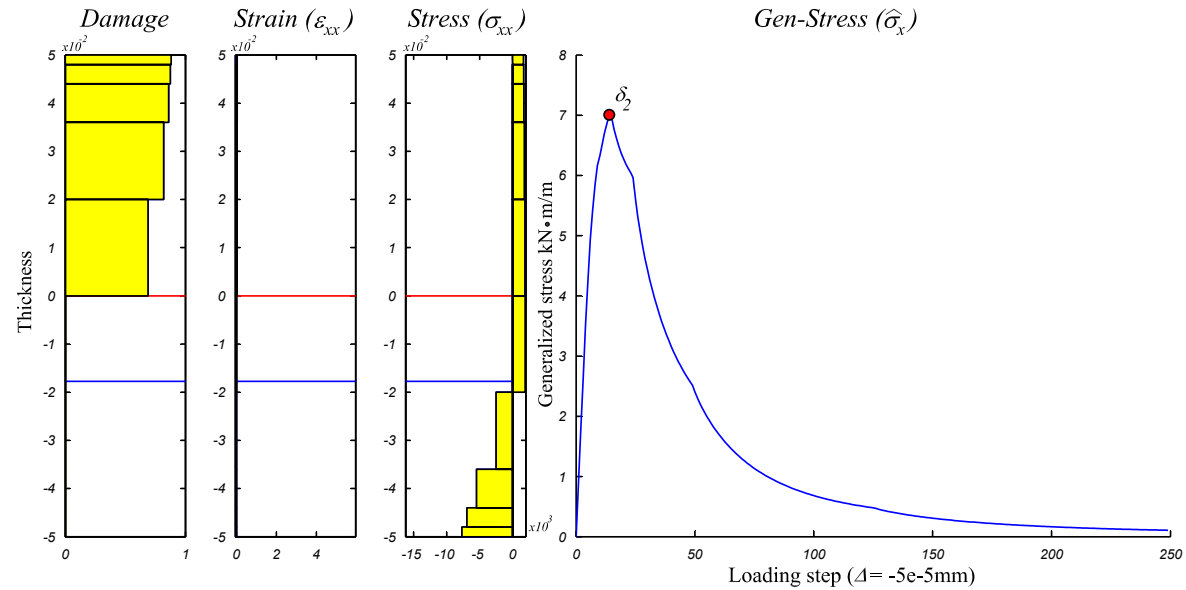

Gen-Stress $\left(\widehat{\sigma}_{x}\right)$

(b) Step 14, $\delta_{2}=0.70 \mathrm{~mm}$
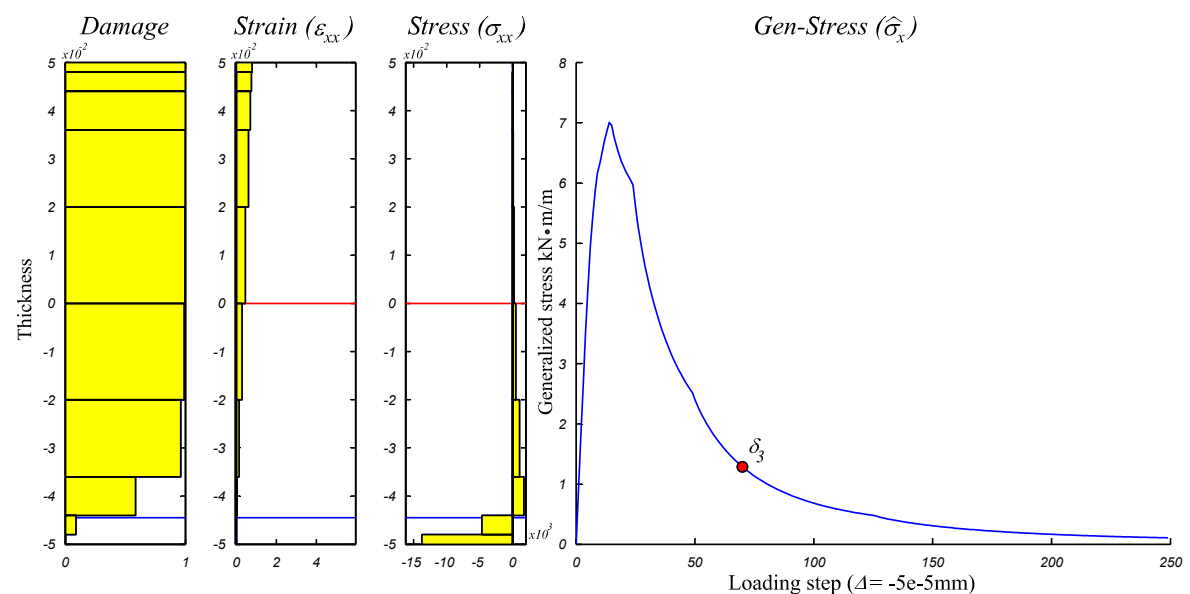

(c) Step 70, $\delta_{3}=3.5 \mathrm{~mm}$

Fig. 14 Evolution of damage, strains and stresses for FE 50 (units: $k N$, meters). 


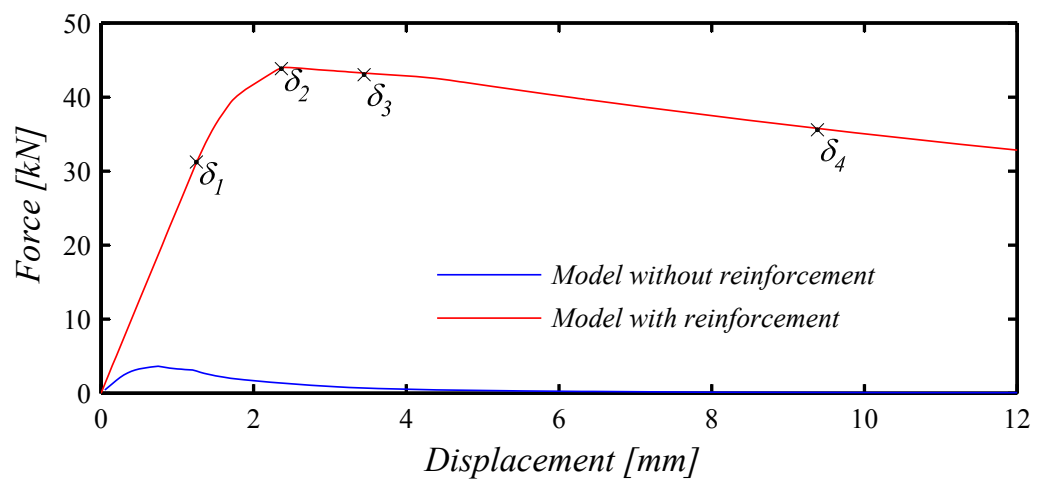

(a) Load-displacement comparison.

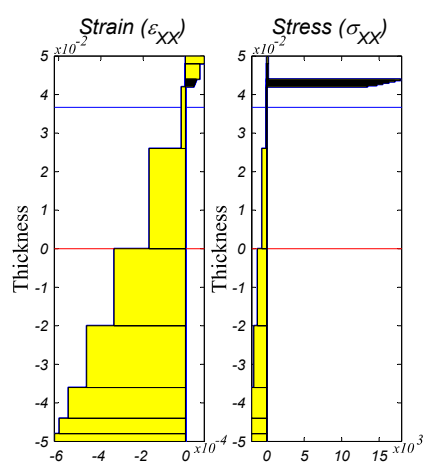

(b) $\delta_{1}=1.25 \mathrm{~mm}$

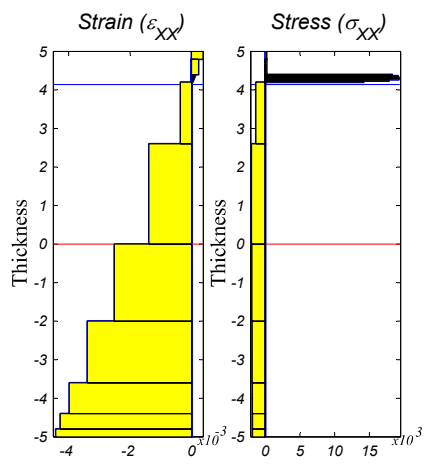

(d) $\delta_{3}=3.45 \mathrm{~mm}$

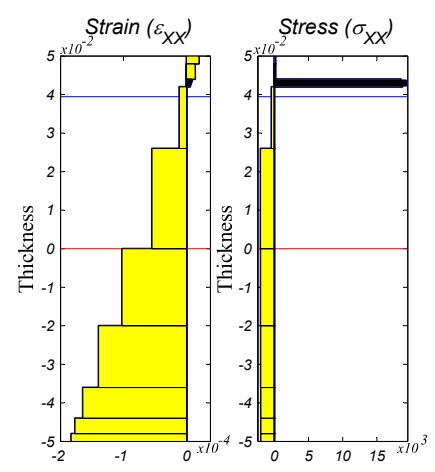

(c) $\delta_{2}=2.30 \mathrm{~mm}$

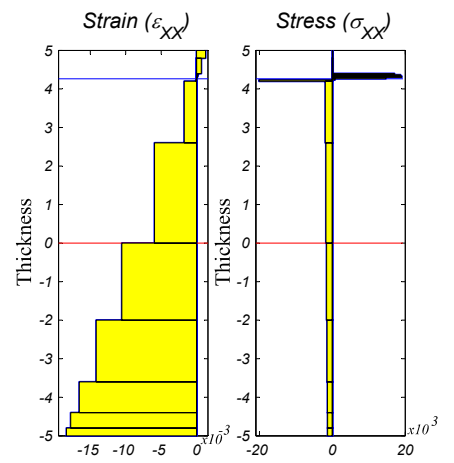

(e) $\delta_{4}=9.40 \mathrm{~mm}$

Fig. 15 Evolution of strains and stresses F.E. 50, for a plate with steel reinforcement (units: kN, meters). 


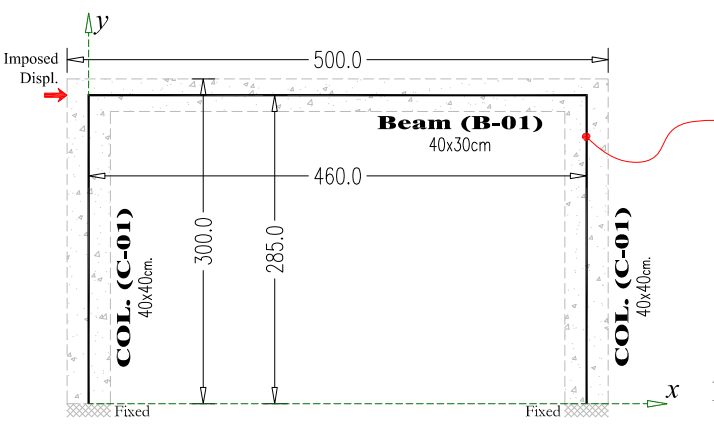

(a)

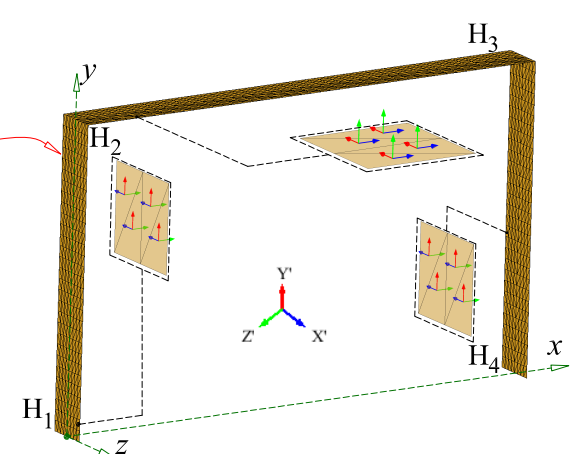

(b)

Fig. 16 Front elevation of typical concrete frame.

\begin{tabular}{c|ccccccccccc} 
Layer & $\mathbf{1}$ & $\mathbf{2}$ & $\mathbf{3}$ & $\mathbf{4}$ & $\mathbf{5}$ & $\mathbf{6}$ to $\mathbf{1 5}$ & $\mathbf{1 6}$ & $\mathbf{1 7}$ & $\mathbf{1 8}$ & $\mathbf{1 9}$ & $\mathbf{2 0}$ \\
\hline $\mathbf{C - 0 1}$ & 1 & 2 & 4 & 8 & 16 & 33.8 & 16 & 8 & 4 & 2 & 1 \\
$\mathbf{B - 0 1}$ & 1 & 2 & 4 & 8 & 16 & 23.8 & 16 & 8 & 4 & 2 & 1
\end{tabular}

Table 1 Tickness of layers (in millimetres) for concrete elements.

modelled as laminate materials combining ROM and CDM. Also, the analysis can be performed without high amount of computational resources, since it is not expensive to add more layers in order to make a more refined analysis.

Some other representative examples have been chosen as a comparison, although the full potential of the element presented here, can only be noticed using structures computationally expensive to reproduce due to the meshing requirements. To reproduce next examples of this section, the proposed scheme has been implemented into a DKT-OPT shell element [39], which is a triangular flat element with 18 D.O.F and with drilling rotation. The arrange of the D.O.F used by the DKT-OPT is depicted in the vector of equation 35.

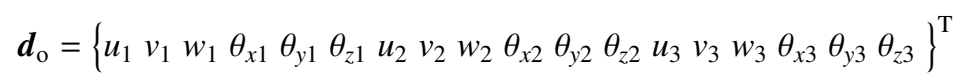

\subsection{Unreinforced concrete frame}

Practical examples presented in current section (4.4) and in the following (4.5) have been originally proposed by Molina et al in [40]. These correspond to numerical analyses made with solid 3D finite elements and will be used as reference for comparison purposes, in order to show the potential of the proposed scheme.

An unreinforced concrete frame is presented, in which it is intended to reproduce the mechanical response exhibited by a brittle material. Geometry of the frame is shown in figure 16.a, where dimensions are presented in centimetres. For both cases, width of column C-01 and beam B-01 is 40 centimetres. In both cases, the discretization along the thickness has been done using the dimensions depicted in table 1 for the columns and the beams.

Figure 16.b shows a perspective of the mesh used, and the local bases used for the finite elements. In figure 16.b it is also depicted, as a future reference, the hinges the structure will undergo during the loading process. So, hinges $H_{1}$ and $H_{4}$ are the ones at the bottom of the columns, whereas hinges $H_{2}$ and $H_{3}$ are expected to occur at the beam ends.

The present analysis has been performed using two loading phases, the first one correspond to the selfweight loading condition, and the second one, corresponds to a push-over analysis, which is defined with an horizontal monotonically increased displacement, imposed directly in the FE at the left top of the frame (figure 16.a) 
There is only one simple material used to model the current frame, it corresponds to concrete, whose mechanical properties agree with those used by Molina et al in [40], and for convenience have been reproduced below.

- Elasticity modulus: $E_{x x}=E_{y y}=2.5 \times 10^{4} \mathrm{MPa}$

- Poisson's ratio: $v=0.20$

- Yield criterion: Mohr Coulomb.

- Damage thresholds:

$$
\begin{aligned}
\sigma_{y c} & =30 M P a \\
\sigma_{y t} & =3 M P a
\end{aligned}
$$

- Fracture and crushing energy:

$$
\begin{aligned}
G_{c} & =20.0 \mathrm{kPa} \cdot \mathrm{m} \\
G_{t} & =0.2 \mathrm{kPa} \cdot \mathrm{m}
\end{aligned}
$$

- self-weight $\gamma=2500 \mathrm{~kg} / \mathrm{m}^{3}$

To assess the secant tensor of each layer has been used equation 21 corresponding to an isotropic damage model. Finally, fracture length $l_{c h}$ is defined equal to the root area of the finite element $l_{c h}=\sqrt{A_{e}}$.

Analysing the obtained results, a manifest brittle material behaviour is noticed in the force-displacement response (fig. 17.a). The low performance of the concrete under tensile stresses, and the absence of reinforcing steel on it, conditioned a poor overall efficiency of the structural system. In order to carry out a proper description of the obtained displacement-force response graph, it has been divided into 6 segments and detailed in next lines. The values of the imposed displacements $\delta_{1}$ to $\delta_{6}$ with theirs corresponding reaction are listed in table 2, whereas the corresponding deformation amplified 100 times are shown in figures 17.b to $17 . \mathrm{g}$.

- Segment $\overline{O \delta_{1}}$, this is where the elastic response occurs, as can be seen, there is a good match among initials stiffnesses obtained with the proposed scheme while compared with the reference solution. This is straightforward since all FE are under an elastic range ( $a=b$ from figure 2).

- Segment $\overline{\delta_{1} \delta_{2}}$, is where non-linear process starts, reaching the highest strength (at $\left.\delta_{2}\right)$ of the concrete frame.

- Segment $\overline{\delta_{2} \delta_{3}}$, shows an abrupt loss of stiffness. It is manifested with the appearance of hinges $H_{1}$ and $\mathrm{H}_{4}$ at the bottom of the columns. At this point no hinge has been formed at the column-beam joints.

- Segment $\overline{\delta_{3} \delta_{4}}$ : another lost of stiffness has occur, more abrupt even than the previous one. Similar to the previous loading segment, the loss of stiffness is produced by the apparition of a new hinge $\left(\mathrm{H}_{3}\right)$.

- Segment $\overline{\delta_{4} \delta_{5}}$, at this loading segment, the structure withstand with only one remaining union rigidly tied.

- Segment $\overline{\delta_{5} \delta_{6}}$, finally structure yields, and the hinge $H_{2}$ is formed. Beyond displacement $\delta_{6}$ it is assumed the structure has lost its whole capacity to withstand lateral forces, which is also confirmed by the deformation undergone (fig 17.g) where all expected hinges have been formed.

As can be seen, beyond displacement $\delta_{1}$ there is not an exact match between the reference solution and the obtained results. However, the efficiency of the proposed scheme is proved with the obtained results since has been possible to reproduce the abrupt degradation at the beam-column joint, and at the support of the columns. 


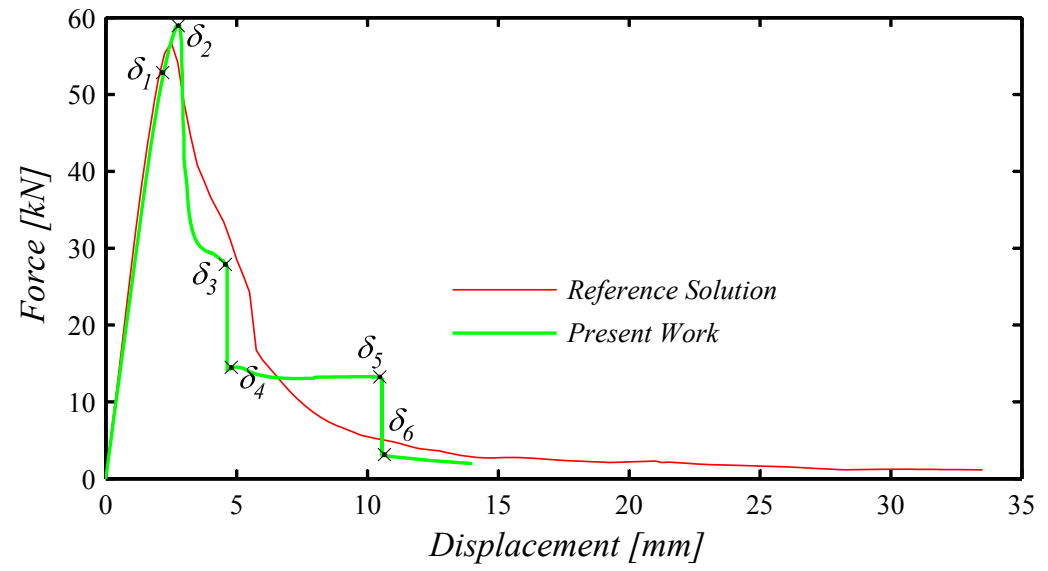

(a) Load-displacement comparison.

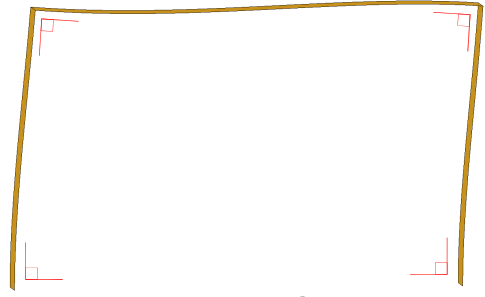

(b) Displ. $\delta_{1}$

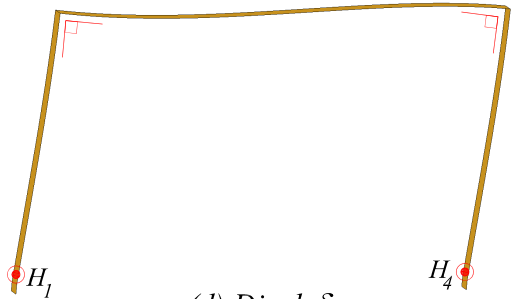

(d) Displ. $\delta_{3}$

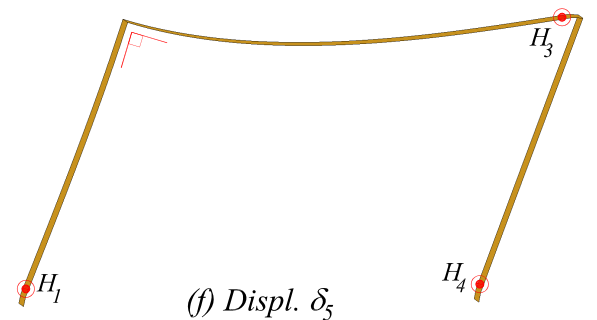

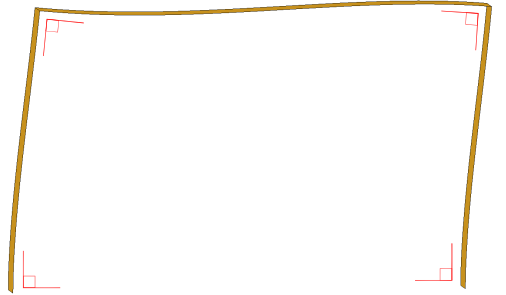

(c) Displ. $\delta_{2}$

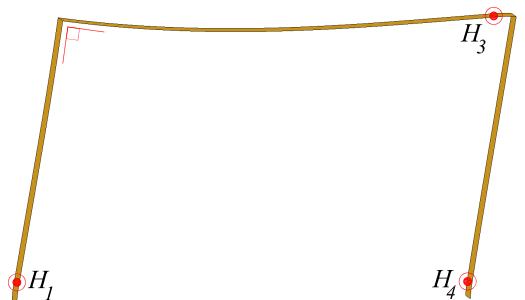

(e) Displ. $\delta_{4}$

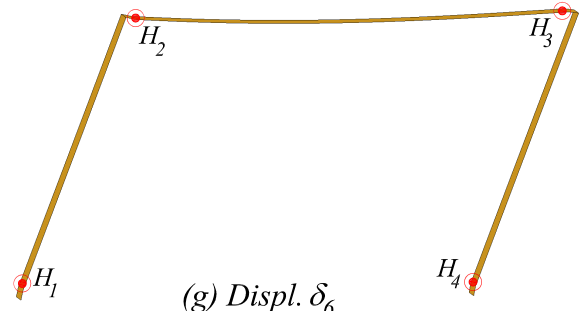

(g) Displ. $\delta_{6}$

Fig. 17 Obtained results for the unreinforced concrete frame. 


\begin{tabular}{ccc}
\hline$\delta$ & Displ. $(\mathrm{mm})$ & Reaction $(\mathrm{N})$ \\
\hline$\delta_{1}$ & 2.11 & $51.11 \times 10^{3}$ \\
$\delta_{2}$ & 2.71 & $58.82 \times 10^{3}$ \\
$\delta_{3}$ & 4.51 & $28.23 \times 10^{3}$ \\
$\delta_{4}$ & 4.55 & $14.22 \times 10^{3}$ \\
$\delta_{5}$ & 10.51 & $13.14 \times 10^{3}$ \\
$\delta_{6}$ & 10.66 & $3.08 \times 10^{3}$ \\
\hline
\end{tabular}

Table 2 Values of representative displacements for figure 17.

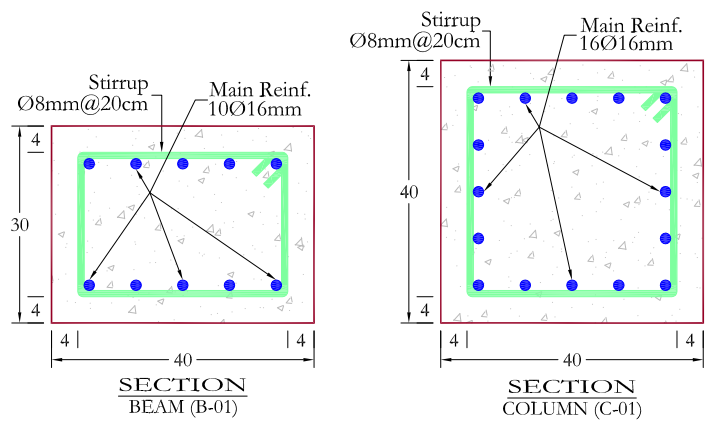

Fig. 18 Cross section of structural elements.

\begin{tabular}{c|ccccccc} 
Layer & $\mathbf{1 , 3 4}$ & $\mathbf{2 , 3 3}$ & $\mathbf{3 , 3 2}$ & $\mathbf{4 , 3 1}$ & $\mathbf{5 , 3 0}$ & $\mathbf{6 - 1 1}, \mathbf{2 4 - 2 9}$ & $\mathbf{1 2 - 2 3}$ \\
\hline $\mathbf{C - 0 1}$ & 2 & 4 & 8 & 16 & 20 & 2.66 & 22.33 \\
$\mathbf{B - 0 1}$ & 2 & 4 & 8 & 16 & 20 & 2.66 & 14.00
\end{tabular}

Table 3 Tickness of layers (in millimetres) for concrete elements. 


\begin{tabular}{ccc}
\hline $\boldsymbol{\delta}$ & Displ. $(\mathrm{mm})$ & Reaction $(\mathrm{N})$ \\
\hline$\delta_{1}$ & 1.84 & $54.55 \times 10^{3}$ \\
$\delta_{2}$ & 11.47 & $171.01 \times 10^{3}$ \\
$\delta_{3}$ & 15.34 & $203.72 \times 10^{3}$ \\
$\delta_{4}$ & 18.10 & $191.60 \times 10^{3}$ \\
\hline
\end{tabular}

Table 4 Values of representative displacements for the RC frame.

\subsection{Reinforced Concrete Frame}

Geometry and mesh for this model are similar to the one presented in section 4.4, i.e, they correspond to figure 16. Also, loading conditions are the same, namely, two loading stages have been applied, first a self-weight loading condition, and then, a monotonically increased displacement.

Mechanical properties used for modelling concrete, agree with the used in section 4.4, whereas mechanical properties of steel fibres are reproduced below.

- Elasticity modulus: $E_{x x}=2.1 \times 10^{5} \mathrm{MPa} ; E_{y y}=2.5 \times 10^{4} \mathrm{MPa}$.

- Poisson's ratio: $v=0.00$.

- Yield criterion: Von Mises.

- Damage thresholds:

$$
\begin{aligned}
& \sigma_{y c}=412 M P a \\
& \sigma_{y t}=412 M P a .
\end{aligned}
$$

- Fracture and crushing energy:

$$
\begin{aligned}
G_{c} & =20.0 \mathrm{kPa} \cdot \mathrm{m} \\
G_{t} & =20.0 \mathrm{kPa} \cdot \mathrm{m}
\end{aligned}
$$

- self-weight $\gamma=7845 \mathrm{~kg} / \mathrm{m}^{3}$.

Constitutive model adopted for mechanical behaviour of steel is the one proposed by Oller in [18], where plastic internal variable $\kappa_{p}$ goes from 0 to 1 which suits equation 21 since $d$ is replaced by $\kappa_{p}$. Cross sections of structural elements with steel reinforcement are presented in figure 18. Both sections have been discretized using 34 layers with the thickness distribution shown in table 3. As can be noticed, the layer's thickness has been adjusted to the position of the steel reinforcement.

Figure 19. a displays the force displacement response for both analysis, the one carried out by Molina et al [40] with four-nodded Lagrangian elements, and the one of the present work. From such figure it can be seen a perfect matching between initial stiffness of both models. A slight difference appears for an applied load close to $50 \mathrm{kN}$, when damage in concrete starts (displacement $\delta_{1}$ ).

A higher difference takes place when the steel reinforcement at base of the columns, goes into non-linear range (displacement $\delta_{2}$ ). The difference in the obtained results at point $\delta_{2}$ may be due to the difference in the constitutive theory used to model the non-linear behaviour of the steel. Point $\delta_{3}$ of figure 19.a is where the reinforced concrete frame presents its higher resistance to lateral forces, beyond such point, a slight difference is presented between both results, the tendency depicted infers a plastic process taking place in the steel reinforcement. Finally, beyond imposed displacement $\delta_{4}$ the response of both models are very much alike.

Damage on bottommost layer is shown in figure 19.b whereas figure 19.c depicts the damage on the topmost layer of the laminate material (see normal direction of local $z$ axis on figure 16). The evolution of the damage shows the same initial behaviour as the case studied in section 4.4, namely, it starts at the bottom 
28

C. Escudero et al / Finite Elements in Analysis and Design 00 (2016) 1-31

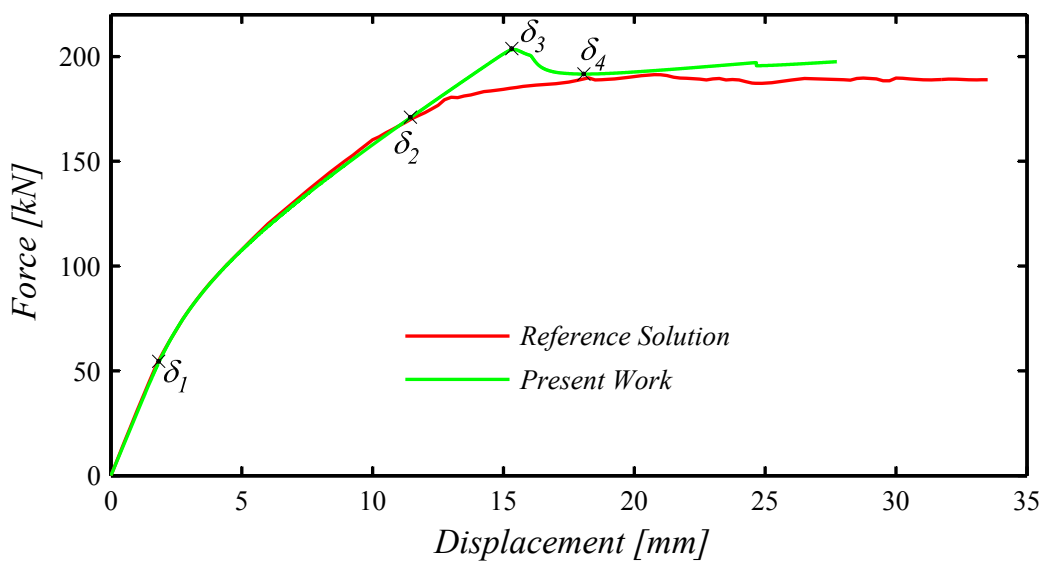

(a) Load-displacement comparison.

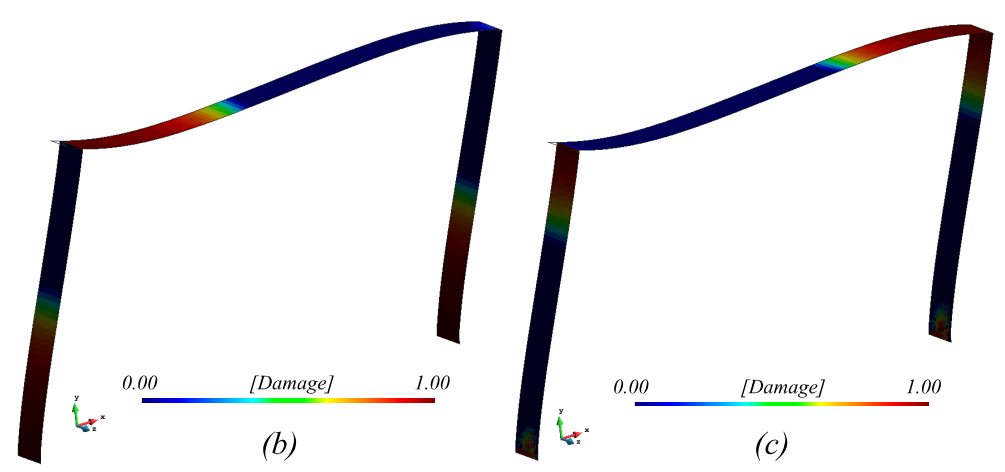

Fig. 19 Obtained results for a reinforced concrete frame. 
of the columns, and then at the beam-column joint, however, due to the presence of steel reinforcement, a most uniform distribution of damaged elements is achieved.

As a conclusion, shall be remarked the fact that, the proper steel reinforcement within the frame, gives the structural system efficiency, in terms of lateral loading resistance and ductility.

\section{Summary and Conclusions}

The starting point of this work has been the use of a 3-node and 2-dimensional triangular shell element with one Gauss point combined with the state-of-the-art rule of mixtures theories for composite materials. Thus it makes it possible to use composite materials whose components can be modelled with non-linear constitutive equations.

In this work has been proposed, developed and implemented in PLCD [41] a laminated shell element able to reproduce the damage due to bending stresses (out-of-plane), besides of the typical damage due to membrane stresses (in-plane), this without being necessary to introduce additional degrees of freedom into its kinematics.

The use of bi-dimensional shell element capable to reproduce not only the damage due membrane stress, but also the damage due to bending stresses, is mandatory to model complex three-dimensional structures. Hence, the idea of this work comes with the necessity of keeping the complexity of the resulting formulation for the FE to a minimum, and consequently, also the required computational resources, thus, make the analysis of three-dimensional structures computationally affordable.

Main conclusions regarding the development of this work are listed below.

- The integration scheme over the thickness proposed in this work, combined with a bi-dimensional triangular element has proved to be capable of reproducing the damage due to bending stresses, and it is not necessary additional degrees of freedom.

- A significant advantage of the modification proposed to the DB-ESL theory, is being able to use laminated materials combined with the state-of-the-art rule of mixtures theories for composites, which in the case of the present work has been applied to model the behaviour of the reinforced concrete confining elements. Also, the use of non-linear constitutive equations to model the behaviour of its components is possible. This makes possible to model and assess the damage of three-dimensional structures with a reasonable low requirement of computational resources.

- To extend the proposed scheme to a composite material is only required to obtain the secant constitutive tensor $\mathbf{D}^{\text {sec }}$ of such composite.

- In order to achieve a fully optimized formulation, improve the efficiency of the analysis process, and speed up both solution and convergence, a tangent stiffness matrix for the element have to be formulated or evaluated using perturbations techniques.

- Although in this work good results have been obtained modelling beams and columns as 2DFEs with the modification proposed to the DB-ESL approach, such examples were used for comparative purposes, and to show the efficiency of the scheme. This modelling strategy is not a common practice, since the nature of DB-ESL approach does not allow to take into account the out-of-plane shear resistance conferred by the stirrups. For that reason, the proposed scheme should be combined with another that allows to overcome this situation, or has to be used with caution in the damage assessment of structural elements such as beams of columns when the out-of-plane steel reinforcement play an important role.

\section{Acknowledgements}

This work has been supported by the European Union's Seventh Framework Programme managed by REA under grant agreement 606242: HYPERMEMBRANE-DEMO Demonstration of an adaptable structure for 
architecture applications, by European Research Council through of Advanced Grant: ERC-2012-AdG 320815 COMP-DES-MAT Advanced tools for computational design of engineering materials, and by the the Mexican government through the grant provided by CONACyT to complete the PhD studies. All this support is gratefully acknowledged.

\section{References}

[1] E. Carrera, Theories and finite elements for multilayered, anisotropic, composite plates and shells, Archives of Computational Methods in Engineering 9 (2) (2002) 87-140.

[2] S. Oller, J. Miquel Canet, F. Zalamea, Composite material behavior using a homogenization double scale method, Journal of engineering mechanics 131 (1) (2005) 65-79.

[3] E. Sanchez-Palencia, A. Zaoui, Homogenization techniques for composite media, in: Homogenization Techniques for Composite Media, Vol. 272, 1987.

[4] A. Eijo, Finite element modelling of delamination in advanced composite beams and plates using one- and two-dimensional finite elements based on the refined zigzag theory., PhD dissertation, Escola Tècnica Superior D'Enginyers de Camins, Canals I Ports. Universitat Politècnica de Catalunya, Barcelona, España (2014).

[5] S. Timoshenko, S. Woinowsky-Kringer, Teoría de Placas y Láminas, Ediciones Urmo S. A., Bilbao, España, 1970.

[6] R. Mindlin, Influence of rotatory inertia and shear in flexural motions of isotropic elastic plates, Journal of Applied Mechanics 18 (1951) 31-38.

[7] E. Oñate, Cálculo de Estructuras por el Método de Elementos Finitos. Analisis Elástico Lineal, Centro Internacional de Métodos Numéricos en Ingeniería, Barcelona, Espa na, 1995.

[8] J.-L. Batoz, K.-J. Bathe, L.-W. Ho, A study of three-node triangular plate bending elements, International Journal for Numerical Methods in Engineering 15 (1980) 1771-1812.

[9] E. Reissner, The effect of transverse shear deformation on the bending of elastic plates, Journal of applied Mechanics 12 (1945) 69-77.

[10] R. D. Mindlin, Influence of rotary inertia and shear on flexural motions of isotropic elastic plates.

[11] O. Ochoa, J. Reddy, Finite Element Analysis of Composite Laminates, Klawer Academic Publishers, 1992.

[12] J. Chaboche, Continuum damage mechanics: Part i-general concepts, Journal of Applied Mechanics 55 (1) (1988) 59-64.

[13] J. Chaboche, Continuum damage mechanics: Part ii-damage growth, crack initiation, and crack growth, Journal of Applied Mechanics 55 (1) (1988) 65-72

[14] J. Simo, J. Ju, Strain-and stress-based continuum damage models i. formulation, Mathematical and Computer Modelling 12 (3) (1989) 378.

[15] J. Simo, J. Ju, Strain-and stress-based continuum damage models ii. computational aspects, International journal of solids and structures 23 (7) (1987) 841-869.

[16] J. Oliver, M. Cervera, S. Oller, J. Lubliner, Isotropic damage models and smeared crack analysis of concrete, in: Second international conference on computer aided analysis and design of concrete structures, Vol. 2, 1990, pp. 945-958.

[17] S. Oller, Nonlinear dynamics of structures, CIMNE-Springer, Barcelona, España, 2014

[18] S. Oller, Un modelo de daño continuo para materiales friccionales, Ph.D. thesis, Escola Tècnica Superior D'Enginyers de Camins, Canals I Ports. Universitat Politècnica de Catalunya, Barcelona, España (1998).

[19] L. Jason, A. Huerta, G. Pijaudier-Cabot, S. Ghavamian, An elastic plastic damage formulation for concrete: Application to elementary tests and comparison with an isotropic damage model, Computer methods in applied mechanics and engineering 195 (52) (2006) 7077-7092.

[20] J. Lubliner, J. Oliver, S. Oller, E. Oñate, A plastic-damage model for concrete, International Journal of Solids and Structures 25 (3) (1989) 299-326.

[21] J. Mazars, G. Pijaudier-Cabot, Continuum damage theory-application to concrete, Journal of Engineering Mechanics 115 (2) (1989) 345-365.

[22] X. Tao, D. V. Phillips, A simplified isotropic damage model for concrete under bi-axial stress states, Cement and Concrete Composites 27 (6) (2005) 716-726.

[23] J. A. Paredes, Modelización numérica del comportamiento constitutivo del daño local y global y su correlación con la evolución de las frecuencias naturales en estructuras de hormigón reforzado, PhD dissertation, Escola Tècnica Superior D’Enginyers de Camins, Canals I Ports. Universitat Politècnica de Catalunya, Barcelona, España (2013).

[24] R. Faria, J. Oliver, M. Cervera, A strain-based plastic viscous-damage model for massive concrete structures, International Journal of Solids and Structures 35 (14) (1998) 1533-1558.

[25] J. A. Paredes, A. H. Barbat, S. Oller, A compression-tension concrete damage model, applied to a wind turbine reinforced concrete tower, Engineering Structures 33 (12) 3559-3569.

[26] C. Truesdell, R. Toupin, The classical field theories, Springer, 1960.

[27] F. Rastellini, S. Oller, O. Salomón, E. Oñate, Composite materials non-linear modelling for long fibre-reinforced, Computers \& Structures 86 (2008) 879-896.

[28] F. G. Rastellini, Modelación numérica de la no-linealidad constitutiva de laminados compuestos, Ph.D. thesis, Escola Tècnica Superior D'Enginyers de Camins, Canals I Ports. Universitat Politècnica de Catalunya, Barcelona, España (2006).

[29] X. Martinez, S. Oller, F. Rastellini, A. Barbat, A numerical procedure simulating rc structures reinforced with frp using the serial/parallel mixing theory, Computers \& Structures 86 (2008) 1604-1618. 
[30] M. Molina, S. Oller, A. H. Barbat, X. Martınez, Análisis numérico de estructuras de hormigón reforzadas con frp por medio de la teoría de mezclas serie/paralelo, Revista internacional de métodos numéricos para cálculo y diseño en ingeniería 26 (2) (2010) $135-155$.

[31] M. Cervera, M. Chiumenti, Smeared crack approach: back to the original track, International journal for numerical and analytical methods in geomechanics 30 (12) (2006) 1173-1199.

[32] J. Oliver, A consistent characteristic length for smeared cracking models, International Journal for Numerical Methods in Engineering 28 (2) (1989) 461-474.

[33] S. Oller, Fractura Mecánica, un Enfoque Global, Centro Internacional de Métodos Numéricos en Ingeniería, Barcelona, España, 2001.

[34] J. Oliver, A. Huespe, M. Pulido, E. Chaves, From continuum mechanics to fracture mechanics: the strong discontinuity approach, Engineering Fracture Mechanics 69 (2) (2002) 113-136.

[35] M. Cervera, M. Chiumenti, Mesh objective tensile cracking via a local continuum damage model and a crack tracking technique, Computer methods in applied mechanics and engineering 196 (1) (2006) 304-320.

[36] X. Martinez, S. Oller, E. Barbero, Mechanical response of composites, Chapter: Study of delamination in composites by using the serial/parallel mixing theory and a damage formulation.

[37] J. Martínez García, S. H. Oller Martínez, L. G. Barbu, H. A. Barbat Barbat, Analysis of ultra low cycle fatigue problems with the barcelona plastic damage model, in: Computational Plasticity XII. Fundamentals and Applications, International Center of Numerical Methods in Engineering (CIMNE), 2013, pp. 352-363.

[38] X. Martínez, Micro-mechanical simulation of composite materials using the serial/parallel mixing theory, Ph.D. thesis, Escola Tècnica Superior D’Enginyers de Camins, Canals I Ports. Universitat Politècnica de Catalunya, Barcelona, España (2008).

[39] P. Khosravi, R. Ganesan, R. Sedaghati, Corotational non-linear analysis of thin plates and shells using a new shell element, International Journal for Numerical Methods in Engineering 69 (2007) 859-885.

[40] M. Molina Herrera, S. H. Oller Martínez, H. A. Barbat Barbat, X. Martínez, Estudio de estructuras de hormigón reforzadas con frp mediante la teoría de mezclas serie/paralelo, Revista internacional de Ingenieria de estructuras 13 (1) (2009) 29-54.

[41] CIMNE, PLCd - Non-linear thermomechanic finite element code (http://www.cimne.com/PLCd), International Center of Numerical Methods in Engineering, Barcelona, España, finite element code oriented to PhD student education (1991-2014). 